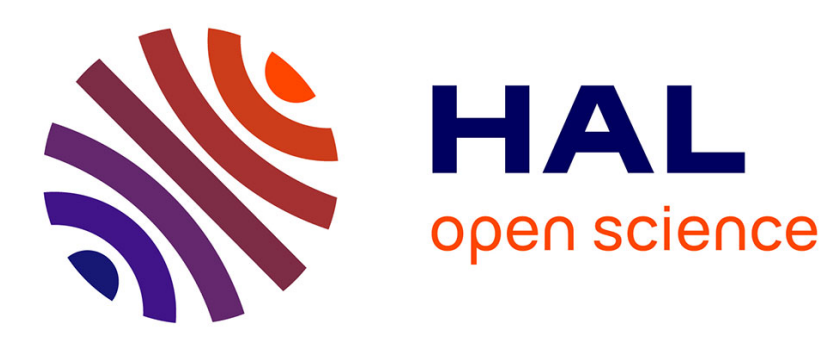

\title{
Factorization Method for Electromagnetic Inverse Scattering from Biperiodic Structures
}

Armin Lecheiter, Dinh Liem Nguyen

\section{To cite this version:}

Armin Lecheiter, Dinh Liem Nguyen. Factorization Method for Electromagnetic Inverse Scattering from Biperiodic Structures. 2012. hal-00762939

\section{HAL Id: hal-00762939 \\ https://hal.science/hal-00762939}

Preprint submitted on 9 Dec 2012

HAL is a multi-disciplinary open access archive for the deposit and dissemination of scientific research documents, whether they are published or not. The documents may come from teaching and research institutions in France or abroad, or from public or private research centers.
L'archive ouverte pluridisciplinaire HAL, est destinée au dépôt et à la diffusion de documents scientifiques de niveau recherche, publiés ou non, émanant des établissements d'enseignement et de recherche français ou étrangers, des laboratoires publics ou privés. 


\title{
Factorization Method for Electromagnetic Inverse Scattering from Biperiodic Structures
}

\author{
Armin Lechleiter* Dinh-Liem Nguyen ${ }^{\dagger}$
}

December 9, 2012

\begin{abstract}
This paper is concerned with the inverse scattering problem of electromagnetic waves from penetrable biperiodic structures in three dimensions. We study the Factorization method as a tool for reconstructing the periodic media from measured data consisting of scattered electromagnetic waves for incident plane electromagnetic waves. We propose a rigorous analysis for the method. A simple criterion is provided to reconstruct the biperiodic structures. We also provide three-dimensional numerical experiments to indicate the performance of the method.
\end{abstract}

\section{Introduction}

We consider inverse scattering of electromagnetic waves from penetrable biperiodic structures in three dimensions. By biperiodic, we mean that the structure is periodic in the, say, $x_{1}$ - and $x_{2}$-direction, while it is bounded in the $x_{3}$ direction. The inverse problem that we treat in this paper is the shape reconstruction of a biperiodic medium from measured data consisting of scattered electromagnetic waves. We consider plane electromagnetic waves as incident fields. The problem that we study here is motivated by the important applications of periodic structures in optics. Applications include diffractive optical filters and organic light-emitting diodes, and non-destructive testing is an important issue to guarantee the functioning of such devices.

Inverse scattering from periodic structures has been an active field of research in the last years. Uniqueness theorems for determining periodic scattering objects from the knowledge of scattered fields can be found in e.g. $[1,8,9,17,32]$. In the general context of acoustic and electromagnetic inverse scattering, qualitative methods has been received much considerable attentions, see Chapter 5 of [20]. Among those methods, the most developed is the linear sampling method which was first introduced in [13] for the scalar case of obstacle inverse scattering. It aims to compute a picture of the shape of the scattering object from measured data. Since the method is relatively rapid and does not need a-priori knowledge, it has attracted much research in recent years. One can find recent developments of the linear sampling method in [10,11]. The linear sampling method has been extended to inverse scattering involving periodic media, in $[15,16,33]$. However, in spite of the advantages of the method, a full mathematical justification still remains open, see [10]. Some results on the justification of the linear sampling method have been recently obtained in $[3,6]$.

As an attempt to improve the linear sampling method, the so-called Factorization method has been developed in $[18,21]$. The latter method has rigorous justification, keeps the previous advantages and of course is an interesting tool for reconstruction problems in inverse scattering. However, there is only a restricted class of scattering problems to which the Factorization method can be applied, see [20]. Recently this method has been extended to periodic inverse scattering problems. In $[4,5]$ the authors studied the Factorization method for the imaging problem of impenetrable periodic structures with Dirichlet and impedance boundary

\footnotetext{
${ }^{*}$ Center for Industrial Mathematics, University of Bremen, 28359 Bremen, Germany
}

${ }^{\dagger}$ DEFI, INRIA Saclay-Ile-de-France and Ecole Polytechnique, 91128 Palaiseau, France 
conditions. The paper [23] considered imaging of penetrable periodic interfaces between two dielectrics in two dimensions.

In the present work we aim to study the Factorization method as a tool for reconstructing three dimensional biperiodic structures for data consisting of scattered electromagnetic waves. More specific, the measured data that we consider here are the coefficients of evanescent and propagating modes of the scattered fields in the radiation condition. Given those coefficients of tangential components of the electromagnetic scattered fields, the inverse problem is then to determine the three-dimensional penetrable biperiodic scatterer. As presented in the rest of the paper, the Factorization method is shown to be an efficient tool to our imaging problem. From a full mathematical justification of the method, a simple criterion for imaging is shown to work accurately in the three-dimensional numerical experiments which, to the best of our knowledge, are the first numerical examples for this method in a biperiodic setting.

Our analysis extends approaches in $[4,20,22]$ to Maxwell's equations in a biperiodic setting. We adapt the special plane incident fields introduced in [4] for the periodic scalar case to the vectorial problem, which allows us to suitably factorize the near field operator. Further, a modified version of the method studied in [22] treats the case that the imaginary part of the middle operator in the factorization is just semidefinite. Since this generalization is of some importance for the problem under consideration, we give a complete proof. Finally the necessary properties of the middle operator are obtained by the approach in [20] for obstacle inverse scattering of electromagnetic waves.

The paper is organized as follows: In Section 2 we introduce the direct problem and set up the corresponding inverse problem. Section 3 is dedicated to study the factorization of the near field operator. Section 4 contains an abstract result on range identity theorem with complete proof. We derive the necessary properties of the middle operator in the factorization in Section 5 while a characterization of the biperiodic structure is given in Section 6. Finally, section 7 is devoted to numerical experiments to examine the performance of the method

\section{Problem Setting}

We consider scattering of time-harmonic electromagnetic waves from a biperiodic structure. The electric field $E$ and the magnetic field $H$ are governed by the time-harmonic Maxwell equations at frequency $\omega>0$ in $\mathbb{R}^{3}$,

$$
\begin{aligned}
\operatorname{curl} H+\mathrm{i} \omega \varepsilon E & =\sigma E \quad \text { in } \mathbb{R}^{3}, \\
\operatorname{curl} E-\mathrm{i} \omega \mu_{0} H & =0 \quad \text { in } \mathbb{R}^{3} .
\end{aligned}
$$

Here the electric permittivity $\varepsilon$ and the conductivity $\sigma$ are real bounded measurable function which are $2 \pi$-periodic in $x_{1}$ and $x_{2}$, and $\mu_{0}$ is the positive constant magnetic permeability. Further, we assume that $\varepsilon$ equals $\varepsilon_{0}>0$ and that $\sigma$ vanishes outside the biperiodic structure. As usual, the problem (1)-(2) has to be completed by a radiation condition that we set up using Fourier series.

Let us denote the relative material parameter by

$$
\varepsilon_{\mathrm{r}}:=\frac{\varepsilon+\mathrm{i} \sigma}{\varepsilon_{0}} .
$$

Note that $\varepsilon_{\mathrm{r}}$ equals 1 outside the biperiodic structure. Recall that the magnetic permeability $\mu_{0}$ is constant which motivates us to work with the divergence-free magnetic field, that is, $\operatorname{div} H=0$. Hence, introducing the wave number $k=\omega\left(\varepsilon_{0} \mu_{0}\right)^{1 / 2}$, and eliminating the electric field $E$ from (1)-(2), we find that

$$
\operatorname{curl}\left(\varepsilon_{\mathrm{r}}^{-1} \operatorname{curl} H\right)-k^{2} H=0 \quad \text { in } \mathbb{R}^{3} .
$$

Now we define that a function $u: \mathbb{R}^{3} \rightarrow \mathbb{C}^{3}$ is called $\alpha$-quasiperiodic if, for $\alpha:=\left(\alpha_{1}, \alpha_{2}, 0\right)$ and $\left(x_{1}, x_{2}, x_{3}\right)^{\top} \in$ 
$\mathbb{R}^{3}$

$$
\begin{aligned}
& u\left(x_{1}+2 \pi, x_{2}, x_{3}\right)=e^{2 \pi \mathrm{i} \alpha_{1}} u\left(x_{1}, x_{2}, x_{3}\right), \\
& u\left(x_{1}, x_{2}+2 \pi, x_{3}\right)=e^{2 \pi \mathrm{i} \alpha_{2}} u\left(x_{1}, x_{2}, x_{3}\right) .
\end{aligned}
$$

Assume that the biperiodic structure is illuminated by $\alpha$-quasiperiodic incident electric and magnetic fields $E^{i}$ and $H^{i}$, respectively, satisfying

$$
\operatorname{curl} H^{i}+\mathrm{i} \omega \varepsilon_{0} E^{i}=0, \quad \operatorname{curl} E^{i}-\mathrm{i} \omega \mu_{0} H^{i}=0 \quad \text { in } \mathbb{R}^{3} .
$$

Simple examples for such $\alpha$-quasiperiodic fields are certain plane waves that we introduce below. We wish to reformulate (3) in terms of the scattered field $H^{s}$, defined by $H^{s}:=H-H^{i}$. Straightforward computations show that curl curl $H^{i}-k^{2} H^{i}=0$, and

$$
\operatorname{curl}\left(\varepsilon_{\mathrm{r}}^{-1} \operatorname{curl} H^{s}\right)-k^{2} H^{s}=-\operatorname{curl}\left(q \operatorname{curl} H^{i}\right) \quad \text { in } \mathbb{R}^{3},
$$

where $q$ is the contrast defined by

$$
q:=\varepsilon_{\mathrm{r}}^{-1}-1 .
$$

Since $\varepsilon_{\mathrm{r}}$ is $2 \pi$-periodic in $x_{1}$ and $x_{2}$, and the right-hand side is $\alpha$-quasiperiodic, we seek for an $\alpha$-quasiperiodic solution $H^{s}$. Hence the problem is reduced to the domain $(0,2 \pi)^{2} \times \mathbb{R}$. We complement this problem by a radiation condition that is set up using Fourier techniques. Since the scattered field $H^{s}$ is $\alpha$-quasiperiodic, the function $e^{-\mathrm{i} \alpha \cdot x} H^{s}$ is $2 \pi$-periodic in $x_{1}$ and $x_{2}$, and can hence be expanded as

$$
e^{-\mathrm{i} \alpha \cdot x} H^{s}(x)=\sum_{n \in \mathbb{Z}^{2}} \hat{H}_{n}\left(x_{3}\right) e^{\mathrm{i}\left(n_{1} x_{1}+n_{2} x_{2}\right)}, \quad x=\left(x_{1}, x_{2}, x_{3}\right)^{\top} \in \mathbb{R}^{3} .
$$

Here the Fourier coefficients $\hat{H}_{n}\left(x_{3}\right) \in \mathbb{C}^{3}$ are defined by

$$
\hat{H}_{n}\left(x_{3}\right)=\frac{1}{4 \pi^{2}} \int_{0}^{2 \pi} \int_{0}^{2 \pi} H^{s}\left(x_{1}, x_{2}, x_{3}\right) e^{-\mathrm{i} \alpha_{n} \cdot x} \mathrm{~d} x_{1} \mathrm{~d} x_{2},
$$

where

$$
\alpha_{n}:=\left(\alpha_{1, n}, \alpha_{2, n}, 0\right)=\left(\alpha_{1}+n_{1}, \alpha_{2}+n_{2}, 0\right) .
$$

We define, for $n \in \mathbb{Z}^{2}$,

$$
\beta_{n}:= \begin{cases}\sqrt{k^{2}-\left|\alpha_{n}\right|^{2}}, & k^{2} \geq\left|\alpha_{n}\right|^{2}, \\ \mathrm{i} \sqrt{\left|\alpha_{n}\right|^{2}-k^{2}}, & k^{2}<\left|\alpha_{n}\right|^{2},\end{cases}
$$

and for some technical reason we assume in the following that

$$
\beta_{n} \neq 0 \quad \text { for all } n \in \mathbb{Z}^{2} .
$$

Recall that $\varepsilon_{\mathrm{r}}^{-1}$ equals one outside the structure. This means $\varepsilon_{\mathrm{r}}^{-1}=1$ and $q=0$ for $\left|x_{3}\right|>h$ where $h>\sup \left\{\left|x_{3}\right|:\left(x_{1}, x_{2}, x_{3}\right)^{\top} \in \operatorname{supp}(q)\right\}$. Thus it holds that $\operatorname{div} H^{s}$ vanishes for $\left|x_{3}\right|>h$, and equation (4) becomes $\left(\Delta+k^{2}\right) H^{s}=0$ in $\left\{\left|x_{3}\right|>h\right\}$. Using separation of variables, and choosing the upward propagating solution, we set up a radiation condition in form of a Rayleigh expansion condition, prescribing that $H^{s}$ can be written as

$$
H^{s}(x)=\sum_{n \in \mathbb{Z}^{2}} \hat{H}_{n}^{ \pm} e^{\mathrm{i}\left(\alpha_{n} \cdot x+\beta_{n}\left|x_{3}-h\right|\right)} \quad \text { for } x_{3} \gtrless \pm h,
$$

where $\left(\hat{H}_{n}^{ \pm}\right)_{n \in \mathbb{Z}^{2}}$ are the Rayleigh sequences given by

$$
\hat{H}_{n}^{ \pm}:=\hat{H}_{n}( \pm h)=\frac{1}{4 \pi^{2}} \int_{0}^{2 \pi} \int_{0}^{2 \pi} H^{s}\left(x_{1}, x_{2}, \pm h\right) e^{-\mathrm{i} \alpha_{n} \cdot x} \mathrm{~d} x_{1} \mathrm{~d} x_{2}, \quad n \in \mathbb{Z}^{2} .
$$


Note that we require that the series in (7) converges uniformly on compact subsets of $\left\{\left|x_{3}\right|>h\right\}$. Further, note that only a finite number of terms in (7) are propagating plane waves which are called propagating modes, the rest are evanescent modes which correspond to exponentially decaying terms.

Denote by $\bar{D}$ the support of the contrast $q$ in one period $\Omega:=(0,2 \pi)^{2} \times \mathbb{R}$. We make an assumption which is necessary for the subsequent factorization frame work.

Assumption 2.1. We assume that the support $D \subset \Omega$ is open and bounded with Lipschitz boundary and that there exists a positive constant $c$ such that $\operatorname{Re}(q) \geq c>0$ and $\operatorname{Im}(q) \leq 0$ almost everywhere in $\Omega$.

Considering a more general source term on the right hand side of (4), we have the following direct problem: Given $f \in L^{2}(D)^{3}$, find $u: \Omega \rightarrow \mathbb{C}^{3}$ in a suitable function space such that

$$
\operatorname{curl}\left(\varepsilon_{\mathrm{r}}^{-1} \operatorname{curl} u\right)-k^{2} u=-\operatorname{curl}(q / \sqrt{|q|} f) \quad \text { in } \Omega,
$$

and $u$ satisfies the Rayleigh expansion condition (7). In the following, a function which satisfies (7) is said to be radiating. It is also seen that if $u$ is a solution of (4) then $u$ solves (8) for the right hand side of $f=\operatorname{curl} H^{i} / \sqrt{|q|}$.

For a variational formulation of the problem, we define, for any Lipschitz domain $\mathcal{O}$,

$$
\begin{aligned}
H(\operatorname{curl}, \mathcal{O}) & =\left\{v \in L^{2}(\mathcal{O})^{3}: \operatorname{curl} v \in L^{2}(\mathcal{O})^{3}\right\}, \\
H_{\text {loc }}\left(\operatorname{curl}, \mathbb{R}^{3}\right) & =\left\{v: \mathbb{R}^{3} \rightarrow \mathbb{C}^{3}:\left.v\right|_{B} \in H(\operatorname{curl}, B) \text { for all balls } B \subset \mathbb{R}^{3}\right\}, \\
H_{\alpha, \text { loc }}(\operatorname{curl}, \Omega) & =\left\{u \in H_{\text {loc }}(\operatorname{curl}, \Omega): u=\left.U\right|_{\Omega} \text { for some } \alpha \text {-quasiperiodic } U \in H_{\text {loc }}\left(\operatorname{curl}, \mathbb{R}^{3}\right)\right\},
\end{aligned}
$$

and

$$
\Omega_{h}=(0,2 \pi)^{2} \times(-h, h) \quad \text { for } h>\sup \left\{\left|x_{3}\right|:\left(x_{1}, x_{2}, x_{3}\right)^{\top} \in \operatorname{supp}(q)\right\},
$$

with boundaries $\Gamma_{ \pm h}:=(0,2 \pi)^{2} \times\{ \pm h\}$. The variational formulation to the problem (8) is to find a radiating solution $u \in H_{\alpha, \text { loc }}(\operatorname{curl}, \Omega)$ such that

$$
\int_{\Omega}\left(\varepsilon_{\mathrm{r}}^{-1} \operatorname{curl} u \cdot \operatorname{curl} \bar{\psi}-k^{2} u \cdot \bar{\psi}\right) \mathrm{d} x=-\int_{\Omega} q / \sqrt{|q|} f \cdot \operatorname{curl} \bar{\psi} \mathrm{d} x,
$$

for all $\psi \in H_{\alpha, \text { loc }}(\operatorname{curl}, \Omega)$ with compact support. Existence and uniqueness of this problem can be obtained for all but possibly a discrete set of wave numbers $k$, see e.g. [7,14,29]. In the sequel we assume that (9) is uniquely solvable for any $f \in L^{2}(D)^{3}$ and fixed $k>0$. Then we define a solution operator $G: L^{2}(D)^{3} \rightarrow$ $\ell^{2}\left(\mathbb{Z}^{2}\right)^{4}$ which maps $f$ to the Rayleigh sequences $\left(\hat{u}_{1, j}^{+}, \hat{u}_{1, j}^{-}, \hat{u}_{2, j}^{+}, \hat{u}_{2, j}^{-}\right)_{j \in \mathbb{Z}^{2}}$ of the first two components of $u \in H_{\alpha, \text { loc }}(\operatorname{curl}, \Omega)$, solution to $(9)$. Note that the Rayleigh sequences $\hat{u}_{(1,2), j}^{ \pm}$are given by

$$
\hat{u}_{(1,2), j}^{ \pm}=\frac{1}{4 \pi^{2}} \int_{0}^{2 \pi} \int_{0}^{2 \pi} u_{(1,2)}\left(x_{1}, x_{2}, \pm h\right) e^{-\mathrm{i} \alpha_{j} \cdot x} \mathrm{~d} x_{1} \mathrm{~d} x_{2}, \quad j \in \mathbb{Z}^{2} .
$$

Now we introduce the notation $\tilde{b}=\left(b_{1}, b_{2},-b_{3}\right)^{\top}$ for $b=\left(b_{1}, b_{2}, b_{3}\right)^{\top} \in \mathbb{C}^{3}$. To obtain the data for the factorization method we consider the following $\alpha$-quasiperiodic plane waves

$$
\varphi_{j}^{(l) \pm}=p_{j}^{(l)} e^{\mathrm{i}\left(\alpha_{j} \cdot x+\beta_{j} x_{3}\right)} \pm \tilde{p}_{j}^{(l)} e^{\mathrm{i}\left(\alpha_{j} \cdot x-\beta_{j} x_{3}\right)}, \quad l=1,2, \quad j \in \mathbb{Z}^{2},
$$

where $p_{j}^{(l)}=\left(p_{1, j}^{(l)}, p_{2, j}^{(l)}, p_{3, j}^{(l)}\right) \in \mathbb{C}^{3} \backslash\{0\}$ are polarizations chosen such that, for all $j \in \mathbb{Z}^{2}$,

$$
\begin{aligned}
& \text { i) } p_{j}^{(1)} \times p_{j}^{(2)}=c_{j}\left(\alpha_{1, j}, \alpha_{2, j}, \beta_{j}\right)^{\top}, \quad \text { for } c_{j} \in \mathbb{C} \backslash\{0\} . \\
& \text { ii) }\left|p_{j}^{(1)}\right|=\left|p_{j}^{(2)}\right|=1 .
\end{aligned}
$$


Together with the assumption that $\beta_{j} \neq 0$ for all $j \in \mathbb{Z}^{2}$, such polarizations are linear independent. One possible choice is

$$
p_{j}^{(1)}=\left(0, \beta_{j},-\alpha_{2, j}\right) /\left(\left|\beta_{j}\right|^{2}+\alpha_{2, j}^{2}\right)^{1 / 2}, \quad p_{j}^{(2)}=\left(-\beta_{j}, 0, \alpha_{1, j}\right) /\left(\left|\beta_{j}\right|^{2}+\alpha_{1, j}^{2}\right)^{1 / 2} .
$$

Note that $\varphi_{j}^{(l) \pm}$ are hence divergence-free functions for all $j \in \mathbb{Z}^{2}, l=1,2$. Due to the linearity of the problem, a linear combination of several incident fields will lead to a corresponding linear combination of the resulting scattered fields. We obtain such linear combination using sequences $\left(a_{j}\right)_{j \in \mathbb{Z}^{2}}=$ $\left(a_{j}^{(1)+}, a_{j}^{(1)-}, a_{j}^{(2)+}, a_{j}^{(2)-}\right)_{j \in \mathbb{Z}^{2}} \in \ell^{2}\left(\mathbb{Z}^{2}\right)^{4}$ and define the corresponding operator by

$$
H\left(a_{j}\right)=\sqrt{|q|} \sum_{j \in \mathbb{Z}^{2}} \frac{1}{\beta_{j} w_{j}}\left[a_{j}^{(1)+} \operatorname{curl} \varphi_{j}^{(1)+}+a_{j}^{(2)+} \operatorname{curl} \varphi_{j}^{(2)+}+a_{j}^{(1)-} \operatorname{curl} \varphi_{j}^{(1)-}+a_{j}^{(2)-} \operatorname{curl} \varphi_{j}^{(2)-}\right]
$$

where

$$
w_{j}:= \begin{cases}\mathrm{i}, & k^{2}>\alpha_{j}^{2}, \\ \exp \left(-\mathrm{i} \beta_{j} h\right), & k^{2}<\alpha_{j}^{2}\end{cases}
$$

Note that we divide by $\beta_{j} w_{j}$ to make later computations easier.

In our inverse problem the data that we measure are the Rayleigh sequences defined in (10). We know that only the propagating modes are measurable far away from the structure. However, it follows from [17] that we need all the modes to be able to uniquely determine the periodic structure. Hence the operator that models measurements from the periodic inhomogeneous medium of scattered fields caused by the incident fields (14) is referred to be the near field operator, denoted by $N$. We define $N: \ell^{2}\left(\mathbb{Z}^{2}\right)^{4} \rightarrow \ell^{2}\left(\mathbb{Z}^{2}\right)^{4}$ to map a sequence $\left(a_{j}\right)_{j \in \mathbb{Z}^{2}}$ to the Rayleigh sequences of the first two components of the scattered field generated by the incident field $H\left(a_{j}\right)$ defined in (14), i.e.

$$
\left[N\left(a_{j}\right)\right]_{n}:=\left(\hat{u}_{1, n}^{+}, \hat{u}_{1, n}^{-}, \hat{u}_{2, n}^{+}, \hat{u}_{2, n}^{-}\right)_{n \in \mathbb{Z}^{2}},
$$

where $u \in H_{\alpha, \text { loc }}(\operatorname{curl}, \Omega)$ is the radiating solution to (9) for the source $f=H\left(a_{j}\right)$. Then from the definition of the solution operator we have

$$
N=G H .
$$

The inverse scattering problem is now to reconstruct the support $\bar{D}$ of the contrast $q=\varepsilon_{\mathrm{r}}^{-1}-1$ when the near field operator $N$ is given. Note that it is not clear yet that $N$ is a bounded linear operator, but we will prove this in the next section.

\section{Factorization of the Near Field Operator}

We study the inverse problem of the previous section using the factorization method. One of the important steps of the latter method that this section is devoted to is factorizing the near field operator. Before doing that, in the next lemma, we show some properties of the operator $H: \ell^{2}\left(\mathbb{Z}^{2}\right)^{4} \rightarrow L^{2}(D)^{3}$ and its adjoint $H^{*}$. We need the sequence

$$
w_{j}^{*}:= \begin{cases}\exp \left(-\mathrm{i} \beta_{j} h\right), & k^{2}>\alpha_{j}^{2} \\ \mathrm{i}, & k^{2}<\alpha_{j}^{2}\end{cases}
$$

Lemma 3.1. For $p_{j}^{(l)}=\left(p_{1, j}^{(l)}, p_{2, j}^{(l)}, p_{3, j}^{(l)}\right), j \in \mathbb{Z}^{2}, l=1,2$, defined as in (12) and (13), the operator $H$ : $\ell^{2}\left(\mathbb{Z}^{2}\right)^{4} \rightarrow L^{2}(D)^{3}$ is compact and injective, and its adjoint $H^{*}: L^{2}(D)^{3} \rightarrow \ell^{2}\left(\mathbb{Z}^{2}\right)^{4}$ satisfies

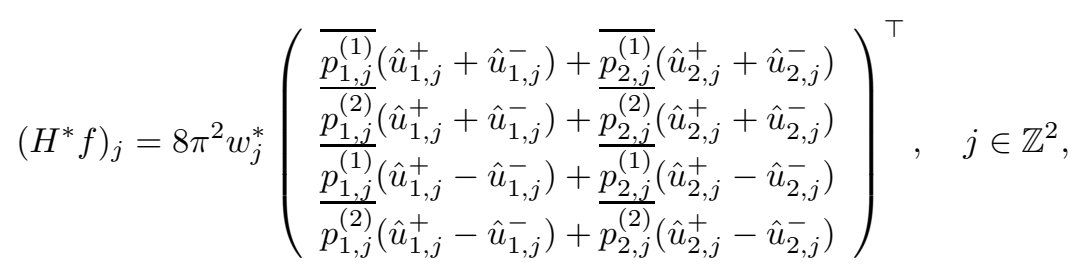


where $\left(\hat{u}_{1, j}^{+}, \hat{u}_{1, j}^{-}, \hat{u}_{2, j}^{+}, \hat{u}_{2, j}^{-}\right)_{j \in \mathbb{Z}^{2}}$ are the Rayleigh sequences of the first two components of $u \in H_{\alpha, l o c}(\operatorname{curl}, \Omega)$, the radiating variational solution to $\operatorname{curl}^{2} u-k^{2} u=\operatorname{curl}(\sqrt{|q|} f)$ in $\Omega$.

Proof. For $l=1,2$ and $j \in \mathbb{Z}^{2}$, we have

$$
\begin{aligned}
& \int_{D} H\left(a_{j}\right) \bar{f} \mathrm{~d} x=\sum_{j \in \mathbb{Z}^{2}}\left[\sum_{l=1,2} \frac{a_{j}^{(l)+}}{\beta_{j} w_{j}} \int_{D} \sqrt{|q| \bar{f}} \cdot \operatorname{curl} \varphi_{j}^{(l)+} \mathrm{d} x+\sum_{l=1,2} \frac{a_{j}^{(l)-}}{\beta_{j} w_{j}} \int_{D} \sqrt{|q| \bar{f}} \cdot \operatorname{curl} \varphi_{j}^{(l)-} \mathrm{d} x\right] \\
& =\left\langle\left(a_{j}\right),\left(\int_{D} \sqrt{|q|} f \cdot \overline{\left(\frac{\operatorname{curl} \varphi_{j}^{(l)+}}{\beta_{j} w_{j}}\right)} \mathrm{d} x, \int_{D} \sqrt{|q|} f \cdot \overline{\left(\frac{\operatorname{curl} \varphi_{j}^{(l)-}}{\beta_{j} w_{j}}\right)} \mathrm{d} x\right)\right\rangle_{\ell^{2}\left(\mathbb{Z}^{2}\right)^{4}} .
\end{aligned}
$$

Note that the equation $\operatorname{curl}^{2} u-k^{2} u=\operatorname{curl}(\sqrt{|q|} f)$ in $\Omega$ with Rayleigh expansion condition is uniquely solvable for all wave number $k>0$. The Fredholm property can be obtained as in [7, 14,29], and using integral representation formulas from Theorem 3.1 in [28] one shows the uniqueness. Now we define $v_{j}^{(l) \pm}=$ $\varphi_{j}^{(l) \pm} /\left(\beta_{j} w_{j}\right)$ and consider a smooth function $\phi \in C^{\infty}(\mathbb{R})$ such that $\phi=1$ in $(-h, h), \phi=0$ in $\mathbb{R} \backslash \overline{(-2 h, 2 h)}$. Then $\phi v_{j}^{(l) \pm}$ belongs to $H_{\alpha}(\operatorname{curl}, \Omega)$ with compact support in $\left\{\left|x_{3}\right|<2 h\right\}$. Assume that $u \in H_{\alpha, \text { loc }}(\operatorname{curl}, \Omega)$ is the variational radiating solution to $\operatorname{curl}^{2} u-k^{2} u=-\operatorname{curl}(\sqrt{|q|} f)$ in $\Omega$. We have

$$
\begin{array}{r}
\int_{D} \sqrt{|q|} f \cdot \operatorname{curl} \overline{v_{j}^{(l) \pm}} \mathrm{d} x=\int_{\Omega_{h}}\left(\operatorname{curl} u \cdot \operatorname{curl} \overline{v_{j}^{(l) \pm}}-k^{2} u \cdot \overline{v_{j}^{(l) \pm}}\right) \mathrm{d} x \\
+\int_{\Omega_{2 h} \backslash \Omega_{h}}\left(\operatorname{curl} u \cdot \operatorname{curl}\left(\overline{\phi v_{j}^{(l) \pm}}\right)-k^{2} u \cdot \overline{\phi v_{j}^{(l) \pm}}\right) \mathrm{d} x .
\end{array}
$$

Now using Green's theorems and exploiting the fact that $v_{j}^{(l) \pm}$ and $u$ are divergence-free solutions to the Hemholtz equation in $\mathbb{R}^{3}$ and $\Omega \backslash \Omega_{h}$, respectively, we obtain that

$$
\begin{aligned}
& \int_{D} \sqrt{|q|} f \cdot \operatorname{curl} \overline{v_{j}^{(l) \pm}} \mathrm{d} x=\int_{\Gamma_{h}}\left(e_{3} \times \operatorname{curl} u \cdot \overline{v_{j}^{(l) \pm}}-e_{3} \times \operatorname{curl} \overline{v_{j}^{(l) \pm}} \cdot u\right) \mathrm{d} s \\
&+ \int_{\Gamma_{-h}}\left(e_{3} \times \operatorname{curl} \overline{v_{j}^{(l) \pm}} \cdot u-e_{3} \times \operatorname{curl} u \cdot \overline{v_{j}^{(l) \pm}}\right) \mathrm{d} s \\
&=\left(\int_{\Gamma_{h}}-\int_{\Gamma_{-h}}\right)\left[\partial_{3} \overline{v_{2, j}^{(l) \pm}} u_{2}-\partial_{3} u_{2} \overline{v_{2, j}^{(l) \pm}}+\partial_{3} \overline{v_{1, j}^{(l) \pm}} u_{1}-\partial_{3} u_{1} \overline{v_{1, j}^{(l) \pm}}\right] \mathrm{d} s .
\end{aligned}
$$

Note that we have

$$
\begin{array}{r}
\overline{v_{1, j}^{(l)+}}=\overline{\left(\frac{p_{1, j}^{(l)}}{\beta_{j} w_{j}}\right)}\left(e^{\mathrm{i} \beta_{j} x_{3}}+e^{-\mathrm{i} \beta_{j} x_{3}}\right) e^{-\mathrm{i} \alpha_{j} \cdot x}, \\
\partial_{3} \overline{v_{1, j}^{(l)+}}=\mathrm{i} \beta_{j} \overline{\left(\frac{p_{1, j}^{(l)}}{\beta_{j} w_{j}}\right)}\left(e^{\mathrm{i} \beta_{j} x_{3}}-e^{-\mathrm{i} \beta_{j} x_{3}}\right) e^{-\mathrm{i} \alpha_{j} \cdot x} .
\end{array}
$$

Then by straightforward computation we obtain

$$
\int_{\Gamma_{h}}\left(\partial_{3} \overline{v_{1, j}^{(l)+}} u_{1}-\partial_{3} u_{1} \overline{v_{1, j}^{(l)+}}\right) \mathrm{d} s=\sum_{n \in \mathbb{Z}^{2}} \hat{u}_{1, n}^{+} \int_{\Gamma_{h}} e^{\mathrm{i} \alpha_{n} \cdot x}\left[\partial_{3} \overline{v_{1, j}^{(l)+}}-i \beta_{n} \overline{v_{1, j}^{(l)+}}\right] \mathrm{d} s=8 \pi^{2} w_{j}^{*} \bar{p}_{1, j}^{(l)} \hat{u}_{1, j}^{+} .
$$

Similarly we also have

$$
\begin{gathered}
\int_{\Gamma_{h}}\left(\partial_{3} \overline{v_{2, j}^{(l)+}} u_{2}-\partial_{3} u_{2} \overline{v_{2, j}^{(l)+}}\right) \mathrm{d} s=8 \pi^{2} w_{j}^{*} \overline{p_{2, j}^{(l)}} \hat{u}_{2, j}^{+}, \\
\left.\int_{\Gamma_{-h}}\left(\partial_{3} \overline{v_{2, j}^{(l)+}} u_{2}-\partial_{3} u_{2} \overline{v_{2, j}^{(l)+}}+\partial_{3} \overline{v_{1, j}^{(l)+}} u_{1}-\partial_{3} \overline{u_{1}} \overline{v_{1, j}^{(l)+}}\right) \mathrm{d} s=-8 \pi^{2} w_{j}^{*} \overline{\left(p_{1, j}^{(l)}\right.} \hat{u}_{1, j}^{-}+\overline{p_{2, j}^{(l)}} \hat{u}_{2, j}^{-}\right) .
\end{gathered}
$$


Now substituting the last two equations into (17) we derive

$$
\left.\int_{D} \sqrt{|q|} f \cdot \operatorname{curl} \overline{v_{j}^{(l)+}} \mathrm{d} x=8 \pi^{2} w_{j}^{*} \overline{\left(p_{1, j}^{(l)}\right.} \hat{u}_{1, j}^{-}+\overline{p_{2, j}^{(l)}} \hat{u}_{2, j}^{-}+\overline{p_{1, j}^{(l)}} \hat{u}_{1, j}^{+}+\overline{p_{2, j}^{(l)}} \hat{u}_{2, j}^{+}\right) .
$$

Similarly we have

$$
\int_{D} \sqrt{|q|} f \cdot \operatorname{curl} \overline{v_{j}^{(l)-}} \mathrm{d} x=8 \pi^{2} w_{j}^{*}\left(-\overline{p_{1, j}^{(l)}} \hat{u}_{1, j}^{-}-\overline{p_{2, j}^{(l)}} \hat{u}_{2, j}^{-}+\overline{p_{1, j}^{(l)}} \hat{u}_{1, j}^{+}+\overline{p_{2, j}^{(l)}} \hat{u}_{2, j}^{+}\right)
$$

which shows that $H^{*}$ satisfies (16). Next we show the compactness of $H^{*}$. This relies on the operator $W: \ell^{2}\left(\mathbb{Z}^{2}\right)^{4} \rightarrow \ell^{2}\left(\mathbb{Z}^{2}\right)^{4}$ defined by

$$
W\left(\left(a_{l}\right)_{l \in \mathbb{Z}^{2}}\right)=-8 \pi^{2} w_{j}^{*}\left(\begin{array}{l}
\overline{p_{1, j}^{(1)}}\left(a_{j}^{(1)+}+a_{j}^{(1)-}\right)+\overline{p_{2, j}^{(1)}}\left(a_{j}^{(2)+}+a_{j}^{(2)-}\right) \\
\overline{p_{1, j}^{(2)}}\left(a_{j}^{(1)+}+a_{j}^{(1)-}\right)+\overline{p_{2, j}^{(2)}}\left(a_{j}^{(2)+}+a_{j}^{(2)-}\right) \\
\overline{p_{1, j}^{(1)}}\left(a_{j}^{(1)+}-a_{j}^{(1)-}\right)+\overline{p_{2, j}^{(1)}}\left(a_{j}^{(2)+}-a_{j}^{(2)-}\right) \\
\overline{p_{1, j}^{(2)}}\left(a_{j}^{(1)+}-a_{j}^{(1)-}\right)+\overline{p_{2, j}^{(2)}}\left(a_{j}^{(2)+}-a_{j}^{(2)-}\right)
\end{array}\right)^{\top}, \quad j \in \mathbb{Z}^{2} .
$$

Since $\left(w_{j}^{*}\right)_{j \in \mathbb{Z}^{2}}$ is a bounded sequence, and since the sequences $\left(p_{j}^{(l)}\right)_{j \in \mathbb{Z}^{2}}$ are bounded for $l=1,2$ due to (13), the operator $W$ is bounded. Now we define the operator

$$
Q: L^{2}(D)^{3} \rightarrow \ell^{2}\left(\mathbb{Z}^{2}\right)^{4}
$$

which maps $f$ to $\left(\hat{u}_{1, j}^{+}, \hat{u}_{1, j}^{-}, \hat{u}_{2, j}^{+}, \hat{u}_{2, j}^{-}\right)$where $u$ is the radiating variational solution to $\operatorname{curl}^{2} u-k^{2} u=$ $\operatorname{curl}(\sqrt{|q|} f)$ in $\Omega$. Then we have

$$
H^{*}=-W Q .
$$

The following trace spaces are necessary for our proof: We define

$$
Y\left(\Gamma_{ \pm h}\right)=\left\{f \in H^{-1 / 2}\left(\Gamma_{ \pm h}\right)^{3} \mid \text { there exists } u \in H_{\alpha}\left(\operatorname{curl}, \Omega_{h}\right) \text { with } \pm e_{3}^{\top} \times\left. u\right|_{\Gamma_{ \pm h}}=f\right\}
$$

with norm

$$
\|f\|_{Y\left(\Gamma_{ \pm h}\right)} \inf _{u \in H_{\alpha}\left(\operatorname{curl}, \Omega_{h}\right), \pm e_{3}^{\top} \times\left. u\right|_{\Gamma_{ \pm h}}=f}\|u\|_{H_{\alpha}\left(\operatorname{curl}, \Omega_{h}\right)} .
$$

The trace spaces $Y\left(\Gamma_{ \pm h}\right)$ are Banach spaces with this norm, see [26]. In the latter reference one also shows that the operation $u \mapsto\left((0,0, \pm 1) \times\left. u\right|_{\Gamma_{ \pm h}}\right) \times(0,0, \pm 1)$ is bounded from $H_{\alpha}\left(\operatorname{curl}, \Omega_{h}\right)$ into $Y^{\prime}\left(\Gamma_{ \pm h}\right)$ which is the dual space of $Y\left(\Gamma_{ \pm h}\right)$.

Now we know that the operation which maps $f \in L^{2}(D)^{3}$ into $u \in H_{\alpha, \text { loc }}$ (curl, $\Omega$ ), radiating variational solution to $\operatorname{curl}^{2} u-k^{2} u=\operatorname{curl}(\sqrt{|q|} f)$, is bounded. Note that $\left((0,0, \pm 1) \times\left. u\right|_{\Gamma_{ \pm h}}\right) \times(0,0, \pm 1)=\left(u_{1}, u_{2}, 0\right)$. We obtain that the operations $\left.f \mapsto\left(u_{1}, u_{2}, 0\right)\right|_{\Gamma_{h}}$ and $\left.\left(u_{1}, u_{2}, 0\right)\right|_{\Gamma_{h}} \mapsto\left(\hat{u}_{1, j}^{+}, \hat{u}_{2, j}^{+}\right)$are bounded from $L^{2}(D)^{3}$ into $Y^{\prime}\left(\Gamma_{h}\right)$ and from $Y^{\prime}\left(\Gamma_{h}\right)$ into $\ell^{2}\left(\mathbb{Z}^{2}\right)^{2}$, respectively. Similarly for $\Gamma_{-h}$ we obtain that $f \mapsto\left(\hat{u}_{1, j}^{-}, \hat{u}_{2, j}^{-}\right)$are bounded from $L^{2}(D)^{3}$ into $\ell^{2}\left(\mathbb{Z}^{2}\right)^{2}$. Together with the boundedness of the sequence $\left(w_{j}^{*}\right)_{j \in \mathbb{Z}^{2}}, Q$ is a bounded operator. We know that in a neighborhood of $\Gamma_{ \pm h} u$ solves the Helmholtz equation. Hence elliptic regularity results [25] imply that $u$ is $H^{2}$-regular in a neighborhood of $\Gamma_{ \pm h}$, thus, $\left.f \mapsto\left(u_{1}, u_{2}, 0\right)\right|_{\Gamma_{ \pm h}}$ is compact operations from $L^{2}(D)^{3}$ into $Y^{\prime}\left(\Gamma_{ \pm h}\right)$. Then $Q$ is a compact operator and $H^{*}$ is compact. Therefore $H$ is compact as well.

To obtain the injectivity of $H$, we prove that $H^{*}$ has dense range. It is sufficient to prove that $W$ has dense range and all sequences $\left(\left(\delta_{j l}\right)_{l \in \mathbb{Z}^{2}}, 0,0,0\right),\left(0,\left(\delta_{j l}\right)_{l \in \mathbb{Z}^{2}}, 0,0\right),\left(0,0,\left(\delta_{j l}\right)_{l \in \mathbb{Z}^{2}}, 0\right)$ and $\left(0,0,0,\left(\delta_{j l}\right)_{l \in \mathbb{Z}^{2}}\right)$ belong to the range of $Q$ (by definition, the Kronecker symbol $\delta_{j l}$ equals one for $j=l$ and zero otherwise). 
The operator $W$ has dense range due to the fact that

$$
\operatorname{det}\left(\begin{array}{cccc}
\overline{p_{1, j}^{(1)}} & \overline{p_{1, j}^{(1)}} & \overline{p_{2, j}^{(1)}} & \overline{p_{2, j}^{(1)}} \\
\frac{p_{1, j}^{(2)}}{p_{1, j}^{(2)}} & \frac{p_{2, j}^{(2)}}{p_{2, j}^{(2)}} \\
\frac{p_{1, j}^{(1)}}{p_{1, j}^{(2)}} & -\frac{p_{1, j}^{(1)}}{p_{1, j}^{(2)}} & \frac{p_{2, j}^{(1)}}{p_{2, j}^{(2)}} & -\frac{p_{2, j}^{(1)}}{p_{2, j}^{(2)}}
\end{array}\right)=-4\left(\overline{p_{1, j}^{(2)} p_{2, j}^{(1)}}-\overline{p_{2, j}^{(2)} p_{1, j}^{(1)}}\right)^{2}=4\left(\overline{c_{j} \beta_{j}}\right)^{2} \neq 0,
$$

due to the property $(12)$ of the polarizations. Now we show that $\left(\left(\delta_{j l}\right)_{l \in \mathbb{Z}^{2}}, 0,0,0\right)$ belongs to the range of $Q$, and the other cases can be done in a similar way. We choose a cut-off function $\chi_{1, j} \in \mathbb{C}^{\infty}(\mathbb{R})$ such that $\chi_{1, j}(t)=0$ for $t<0$ and $\chi(t)=1$ for $t>h / 2$. Then $\left(x_{1}, x_{2}, x_{3}\right) \mapsto \chi_{1, j}\left(x_{3}\right) \exp \left(\mathrm{i}\left(\alpha_{j} \cdot x+\beta_{j}\left(x_{3}-h\right)\right)\right.$ has Rayleigh sequence $\left(\left(\delta_{j l}\right)_{l \in \mathbb{Z}^{2}}, 0\right)$. For all $j \in \mathbb{Z}^{2}$, we define

$$
\varphi_{j}(x)=\left(\chi_{1, j}\left(x_{3}\right), 0, \chi_{3, j}\left(x_{3}\right)\right)^{\top} \exp \left(\mathrm{i}\left(\alpha_{j} \cdot x+\beta_{j}\left(x_{3}-h\right)\right),\right.
$$

where

$$
\chi_{3, j}\left(x_{3}\right)=-\mathrm{i} \alpha_{1, j} e^{-\mathrm{i} \beta_{j} x_{3}} \int_{0}^{x_{3}} e^{\mathrm{i} \beta_{j} t} \chi_{1, j}(t) \mathrm{d} t .
$$

Then $\operatorname{div} \varphi_{j}=0$ in $\Omega$ and the Rayleigh sequences of the first two components of $\varphi_{j}$ are $\left(\left(\delta_{j l}\right)_{l \in \mathbb{Z}^{2}}, 0,0,0\right)$. Next we show that there exists $f_{j} \in L^{2}(D)^{3}$ such that $\operatorname{curl}^{2} \varphi_{j}-k^{2} \varphi_{j}=\operatorname{curl}\left(\sqrt{|q|} f_{j}\right)$ in $\Omega$ holds in the variational sense. Set

$$
g_{j}(x):=\operatorname{curl}^{2} \varphi_{j}(x)-k^{2} \varphi_{j}(x), \quad x \in \Omega,
$$

then we have $\operatorname{div}\left(g_{j}\right)=0$ in $\Omega$ which also implies that

$$
\int_{\partial \Omega_{h}} g_{j} \cdot \nu \mathrm{d} s=0
$$

Therefore, due to Theorem 3.38 in [26], there exists $\psi_{j} \in H^{1}\left(\Omega_{h}\right)^{3}$ such that

$$
g_{j}=\operatorname{curl} \psi_{j} \quad \text { in } \Omega_{h} .
$$

Define $f_{j}=\sqrt{|q|}^{-1} \psi_{j}$, then $f_{j} \in L^{2}(D)^{3}$ and we have, in the weak sense,

$$
\operatorname{curl}^{2} \varphi_{j}-k^{2} \varphi_{j}=\operatorname{curl}\left(\sqrt{|q|} f_{j}\right) \quad \text { in } \Omega_{h} .
$$

Together with $\operatorname{curl}^{2} \varphi_{j}-k^{2} \varphi_{j}=0$ in $\Omega \backslash \Omega_{h}$, we complete the proof.

Now we show a factorization of the near field operator $N$ in the following theorem. To this end, we define the sign of $q$ by

$$
\operatorname{sign}(q):=\frac{q}{|q|}
$$

Theorem 3.2. Assume that $q$ satisfies the Assumption 2.1. The operator $W$ is defined as in (18). Let $T: L^{2}(D)^{3} \rightarrow L^{2}(D)^{3}$ be defined by $T f=\operatorname{sign}(q)(f+\sqrt{|q|} \operatorname{curl} v)$, where $v \in H_{\alpha, l o c}(\operatorname{curl}, \Omega)$ is the radiating solution to (9). Then the near field operator satisfies

$$
W N=H^{*} T H .
$$

Proof. We recall the operator $Q$ in (19) that maps $f \in L^{2}(D)^{3}$ to the Rayleigh sequences $\left(\hat{u}_{1, j}^{+}, \hat{u}_{1, j}^{-}, \hat{u}_{2, j}^{+}, \hat{u}_{2, j}^{-}\right)$ where $u$ is the radiating variational solution to $\operatorname{curl}^{2} u-k^{2} u=\operatorname{curl}(\sqrt{|q|} f)$ in $\Omega$. By definition of the solution operator $G$ we have $G f=\left(\hat{u}_{1, j}^{+}, \hat{u}_{1, j}^{-}, \hat{u}_{2, j}^{+}, \hat{u}_{2, j}^{-}\right)$where $u \in H_{\alpha, \text { loc }}(\operatorname{curl}, \Omega)$ is a radiating weak solution to $\operatorname{curl}\left(\varepsilon_{\mathrm{r}}^{-1} \operatorname{curl} u\right)-k^{2} u=-\operatorname{curl}(q / \sqrt{|q|} f)$. This means that $\operatorname{curl}^{2} u-k^{2} u=-\operatorname{curl}(\sqrt{|q|} \operatorname{sign}(q)(f+$ $\sqrt{|q|} \operatorname{curl} v)$ ), thus, $G f=-(Q T) f$. Now due to the fact that $N=G H$ we have

$$
W N=W G H=-W Q T H .
$$

Additionally we know from (20) that $H^{*}=-W Q$ which completes the proof. 


\section{The Range Identity Theorem}

This section presents an abstract result on range identities which is necessary to characterize the support $D$ of the contrast $q$. For the convenience of the reader, we give a rather complete proof, see also in [20,22]. First, we introduce real and imaginary part of a bounded linear operator. Let $X \subset U \subset X^{*}$ be a Gelfand triple, that is, $U$ is a Hilbert space, $\mathrm{X}$ is a reflexive Banach space with dual $X^{*}$ for the inner product of $U$, and the embeddings are injective and dense. Then the real and imaginary part of a bounded operator $T: X^{*} \rightarrow X$ are defined in accordance with the corresponding definition for complex numbers,

$$
\operatorname{Re}(T):=\frac{1}{2}\left(T+T^{*}\right), \quad \operatorname{Im}(T):=\frac{1}{2 i}\left(T-T^{*}\right) .
$$

Theorem 4.1. Let $X \subset U \subset X^{*}$ be a Gelfand triple with Hilbert space $U$ and reflexive Banach space $X$. Furthermore, let $V$ be a second Hilbert space and $F: V \rightarrow V, H: V \rightarrow X$ and $T: X \rightarrow X^{*}$ be linear and bounded operators with

$$
F=H^{*} T H
$$

We make the following assumptions:

a) $H$ is compact and injective.

b) There exists $t \in[0,2 \pi]$ such that $\operatorname{Re}\left(e^{\mathrm{it}} T\right)$ has the form $\operatorname{Re}\left(e^{\mathrm{it}} T\right)=T_{0}+T_{1}$ with some positive definite selfadjoint operator $T_{0}$ and some compact operator $T_{1}: X \rightarrow X^{*}$.

c) $\operatorname{Im} T$ is non positive on $X$, i.e., $\langle\operatorname{Im} T \phi, \phi\rangle \leq 0$ for all $\phi \in X$.

Moreover, we assume that one of the two following conditions is fullfilled

d) $T$ is injective and $t$ from $b$ ) does not equal $\pi / 2$ or $3 \pi / 2$.

e) $\operatorname{Im} T$ is negative on the (finite dimensional) null space of $\operatorname{Re}\left(e^{\mathrm{it}} T\right)$, i.e., for all $\phi \neq 0$ such that $\operatorname{Re}\left(e^{\mathrm{i} t} T\right) \phi=$ 0 it holds $\langle\operatorname{Im} T \phi, \phi\rangle<0$.

Then the operator $F_{\sharp}:=\left|\operatorname{Re}\left(e^{\mathrm{it}} F\right)\right|-\operatorname{Im} F$ is positive definite and the ranges of $H^{*}: X^{*} \rightarrow V$ and $F_{\sharp}^{\frac{1}{2}}: V \rightarrow V$ coincide.

Proof. We know that from Theorem 2.15 in [19] it is sufficient to assume that $X=U$ is a Hilbert space and that $H$ has dense range in $U$. The factorization of $F$ implies that $\operatorname{Re}\left(e^{\mathrm{it}} F\right)=H^{*} \operatorname{Re}\left(e^{\mathrm{i} t} T\right) H$ is compact and selfadjoint. By the spectral theorem for such operators, there exists a complete orthonormal eigensystem $\left(\lambda_{j}, \psi_{j}\right)_{j \in \mathbb{N}}$ of $\operatorname{Re}\left(e^{\mathrm{i} t} F\right)$. In consequence, the spaces

$$
V^{+}=\operatorname{span}\left\{\psi_{j}: \lambda_{j}>0\right\} \quad \text { and } \quad V^{-}=\operatorname{span}\left\{\psi_{j}: \lambda_{j} \leq 0\right\}
$$

are invariant under $\operatorname{Re}\left(e^{\mathrm{it}} F\right)$ and satisfy $V=V^{+} \oplus V^{-}$. We set $U^{-}=H V^{-}$.

In the next step we show that $U^{-}$is finite dimensional. The operator $T_{1}=\operatorname{Re}\left(e^{\mathrm{i} t} T\right)-T_{0}$ is a selfadjoint and compact operator, we denote by $\left(\mu_{j}, \phi_{j}\right)_{j \in \mathbb{N}}$ an eigensystem of $T_{1}$. By assumption of $T_{0}$, there exists $\alpha>0$ such that $\left\langle T_{0} \varphi, \varphi\right\rangle \geq \alpha\|\varphi\|^{2}$ for all $\varphi \in U$. We set $W^{+}=\operatorname{span}\left\{\phi_{j}: \mu_{j}>-\alpha\right\}, W^{-}=\operatorname{span}\left\{\phi_{j}: \mu_{j} \leq-\alpha\right\}$ and note that $W^{-}$is finite dimensional since $\mu_{j} \rightarrow 0$. Let now $\phi=H \psi \in U^{-}$with (unique) decomposition $\phi=\phi^{+}+\phi^{-}, \phi^{ \pm} \in W^{ \pm}$. Since $\psi \in V^{-}$,

$$
\begin{aligned}
0 \geq & \left\langle\operatorname{Re}\left(e^{\mathrm{i} t} F\right) \psi, \psi\right\rangle=\left\langle\operatorname{Re}\left(e^{\mathrm{i} t} T\right) H \psi, H \psi\right\rangle=\left\langle\operatorname{Re}\left(e^{\mathrm{i} t} T\right)\left(\phi^{+}+\phi^{-}\right), \phi^{+}+\phi^{-}\right\rangle \\
& =\left\langle\operatorname{Re}\left(e^{\mathrm{i} t} T\right) \phi^{+}, \phi^{+}\right\rangle+\left\langle\operatorname{Re}\left(e^{\mathrm{i} t} T\right) \phi^{-}, \phi^{-}\right\rangle \geq c\left\|\phi^{+}\right\|^{2}-\left\|\operatorname{Re}\left(e^{\mathrm{i} t} T\right)\right\|\left\|\phi^{-}\right\|^{2},
\end{aligned}
$$

thus, $\|\phi\|^{2}=\left\|\phi^{+}\right\|^{2}+\left\|\phi^{-}\right\|^{2} \leq C\left\|\phi^{-}\right\|^{2}$. This shows that the mapping $\phi \mapsto \phi^{-}$is boundedly invertible from $U^{-}$into $W^{-}$. Consequently, $U^{-}$is finite dimensional.

Denseness of the range of $H$ implies that the sum $\overline{H V^{+}}+U^{-}$is dense in $U$. Since $U^{-}$is a finite dimensional and therefore complemented subspace, we can choose a closed subspace $U^{+}$of $\overline{H V^{+}}$such that the (non-orthogonal) sum $U=U^{+} \oplus U^{-}$is direct. Let moreover $U^{0}:=\overline{H V^{+}} \cap U^{-}$be the intersection of $\overline{H V^{+}}$and $U^{-}$, we will show that $U^{0}$ is contained in the kernel of $\operatorname{Re}\left(e^{\mathrm{i} t} T\right)$. We denote $P_{U^{ \pm}}: U \rightarrow U^{ \pm}$the 
canonical projections, that is, every $\phi \in U$ has the unique decomposition $\phi=P_{U^{+}} \phi+P_{U^{-}} \phi$. Both operators $P_{U^{ \pm}}$are bounded and $P_{U^{+}}-P_{U^{-}}$is an isomorphism, since

$$
\left(P_{U^{+}}-P_{U^{-}}\right)^{2}=P_{U^{+}}^{2}+P_{U^{-}}^{2}-P_{U^{+}} P_{U^{-}}-P_{U^{-}} P_{U^{+}}=P_{U^{+}}+P_{U^{-}}=\text {Id } .
$$

From the factorization $\operatorname{Re}\left(e^{\mathrm{it}} F\right)=H^{*} \operatorname{Re}\left(e^{\mathrm{it}} T\right) H$ and the definition of $U^{ \pm}$we obtain that $H^{*} \operatorname{Re}\left(e^{\mathrm{i} t} T\right)\left(U^{-}\right)=$ $\operatorname{Re}\left(e^{\mathrm{it}} F\right)\left(V^{-}\right) \subset V^{-}$. Note also that, by definition we have $U^{+} \subset \overline{H V^{+}}$. In consequence, for $\phi^{-} \in U^{-}$and $\psi^{+} \in V^{+}$we have

$$
0=\left\langle H^{*}\left(\operatorname{Re}\left(e^{\mathrm{i} t} T\right)\right) \phi^{-}, \psi^{+}\right\rangle=\left\langle\operatorname{Re}\left(e^{\mathrm{i} t} T\right) \phi^{-}, H \psi^{+}\right\rangle=\left\langle\phi^{-},\left(\operatorname{Re}\left(e^{\mathrm{i} t} T\right)\right) H \psi^{+}\right\rangle .
$$

We conclude that $\operatorname{Re}\left(e^{\mathrm{i} t} T\right) U^{-} \subset\left(H V^{+}\right)^{\perp}=\left(U^{+} \oplus U^{0}\right)^{\perp} \subset\left(U^{+}\right)^{\perp}$ and, $\operatorname{Re}\left(e^{\mathrm{i} t} T\right) U^{+} \subset \operatorname{Re}\left(e^{\mathrm{i} t} T\right) \overline{H V^{+}} \subset$ $\left(U^{-}\right)^{\perp}$. Indeed, for $\phi^{+} \in \overline{H V^{+}}$there is a sequence $\psi_{n}^{+} \in V^{+}$such that $H \psi_{n}^{+} \rightarrow \phi^{+}$and $\operatorname{Re}\left(e^{\mathrm{i} t} T\right) H \psi_{n}^{+} \subset$ $\left(U^{-}\right)^{\perp}$ by $(21)$, thus, $\operatorname{Re}\left(e^{\mathrm{it} T} T\right) \phi^{+} \subset\left(U^{-}\right)^{\perp}$. For $\phi^{0} \in \overline{H V^{+}} \cap U^{-}$, these mapping properties of $\operatorname{Re}\left(e^{\mathrm{it}} T\right)$ imply that $\operatorname{Re}\left(e^{\mathrm{it}} T\right) \phi^{0}$ is orthogonal both to $U^{-}$and $U^{+}$. Therefore $\operatorname{Re}\left(e^{\mathrm{i} t} T\right) \phi^{0}=0$ and we conclude that $U^{0}=\overline{H V^{+}} \cap U^{-}$is contained in the kernel of $\operatorname{Re}\left(e^{\mathrm{i} t} T\right)$. This inclusion allows to show a factorization of $F_{\sharp}$ in the next step.

Let $\psi \in V$ and $\psi^{ \pm}$be its orthogonal projection on $V^{ \pm}$. Then

$$
\begin{aligned}
\left|\operatorname{Re}\left(e^{\mathrm{i} t} F\right)\right| \psi & =H^{*} \operatorname{Re}\left(e^{\mathrm{i} t} T\right) H\left(\psi^{+}-\psi^{-}\right) \\
& =H^{*} \operatorname{Re}\left(e^{\mathrm{i} t} T\right)\left(P_{U^{+}} H \psi^{+}+P_{U^{-}} H \psi^{+}-P_{U^{+}} H \psi^{-}-P_{U^{-}} H \psi^{-}\right) \\
& =H^{*} \operatorname{Re}\left(e^{\mathrm{i} t} T\right)(P_{U^{+}} H \psi+2 \underbrace{P_{U^{-}} H \psi^{+}}_{\in U^{0} \subset \operatorname{ker}\left(\operatorname{Re}\left(e^{\mathrm{i} t} T\right)\right)}-P_{U^{+}} H \psi) \\
& =H^{*} \operatorname{Re}\left(e^{\mathrm{i} t} T\right)\left(P_{U^{+}}-P_{U^{-}}\right) H \psi
\end{aligned}
$$

This factorization of $\left|\operatorname{Re}\left(e^{\mathrm{it}} F\right)\right|$ yields a factorization of $F_{\sharp}$,

$$
F_{\sharp}=\left|\operatorname{Re}\left(e^{\mathrm{i} t} F\right)\right|-\operatorname{Im} F=H^{*}\left(\operatorname{Re}\left(e^{\mathrm{i} t} T\right)\left(P_{U^{+}}-P_{U^{-}}\right)-\operatorname{Im} T\right) H=H^{*} T_{\sharp} H,
$$

where $T_{\sharp}=\operatorname{Re}\left(e^{\mathrm{i} t} T\right)\left(P_{U^{+}}-P_{U^{-}}\right)-\operatorname{Im} T$. Due to the fact that

$$
\left\langle\operatorname{Re}\left(e^{\mathrm{i} t} T\right)\left(P_{U^{+}}-P_{U^{-}}\right) H \phi, H \phi\right\rangle=\left\langle\left|\operatorname{Re}\left(e^{\mathrm{i} t} F\right)\right| \phi, \phi\right\rangle \geq 0
$$

for all $\phi \in V$ and denseness of the range of $H$ in $U$ we conclude that $\operatorname{Re}\left(e^{\mathrm{it} t} T\right)\left(P_{U^{+}}-P_{U^{-}}\right)$is nonnegative on $U$. Since $T_{\sharp}$ is therefore a nonnegative operator, we can apply the inequality [19, Estimate (4.5)] for bounded nonnegative operators,

$$
\left\langle T_{\sharp} \psi, \psi\right\rangle \geq \frac{1}{\left\|T_{\sharp}\right\|}\left\|T_{\sharp} \psi\right\|^{2}, \quad \psi \in U
$$

Now, we show that assumption d) implies assumption e). Under the assumption d), let $\phi$ belong to the null space of $\operatorname{Re}\left(e^{\mathrm{i} t} T\right)$ and suppose that $\langle\operatorname{Im} T \phi, \phi\rangle=0$. We need to show that this implies that $\phi=0$. By definition of the real part of an operator,

$$
e^{\mathrm{i} t} T \phi+e^{-\mathrm{it}} T^{*} \phi=0
$$

Furthermore, $-\operatorname{Im} T$ is a bounded nonnegative operator so the application of (22) to $-\operatorname{Im} T$ yields

$$
0=\langle-\operatorname{Im} T \phi, \phi\rangle \geq \frac{1}{\|\operatorname{Im} T\|}\|\operatorname{Im} T \phi\|^{2}, \quad \phi \in U,
$$

hence $\|\operatorname{Im} T \phi\|=0$ and $\operatorname{Im} \phi=0$. By definition of the imaginary part, this is to say that $T \phi-T^{*} \phi=0$. Combine this equation with (23) yields that $\left(1+e^{\mathrm{i} 2 t}\right) T \phi=0$. Since $t \in[0,2 \pi] \backslash\left\{\frac{\pi}{2}, \frac{3 \pi}{2}\right\}$, this implies $T \phi=0$ and $\phi=0$ by assumption $\mathrm{d})$. We have hence proven that $\langle\operatorname{Im} T \phi, \phi\rangle<0$ for all $0 \neq \phi \in \operatorname{ker}\left(\operatorname{Re}\left(e^{\mathrm{it}} T\right)\right)$. This is precisely assumption e) which is considered next. 
Assuming e), we will show that $T_{\sharp}$ is injective. Suppose that $T_{\sharp} \phi=0$, then we have $\left\langle\operatorname{Re}\left(e^{\mathrm{i} t} T\right)\left(P_{U^{+}}-\right.\right.$ $\left.\left.P_{U^{-}}\right) \phi, \phi\right\rangle-\langle\operatorname{Im} T \phi, \phi\rangle=0$. Boths terms on the left are nonnegative so we have

$$
\left\{\begin{array}{l}
\left\langle\operatorname{Re}\left(e^{\mathrm{i} t} T\right)\left(P_{U^{+}}-P_{U^{-}}\right) \phi, \phi\right\rangle=0 \\
\langle\operatorname{Im} T \phi, \phi\rangle=0
\end{array}\right.
$$

From this and application of $(22)$ to $\operatorname{Re}\left(e^{\mathrm{i} t} T\right)\left(P_{U^{+}}-P_{U^{-}}\right)$yield $\operatorname{Re}\left(e^{\mathrm{i} t} T\right)\left(P_{U^{+}}-P_{U^{-}}\right) \phi=0$. Moreover, due to the selfadjointness we obtain

$$
\operatorname{Re}\left(e^{\mathrm{i} t} T\right)\left(P_{U^{+}}-P_{U^{-}}\right)=\left(P_{U^{+}}-P_{U^{-}}\right)^{*} \operatorname{Re}\left(e^{\mathrm{i} t} T\right)
$$

and since $P_{U^{+}}-P_{U^{-}}$is an isomorphism so is $\left(P_{U^{+}}-P_{U^{-}}\right)^{*}$. Consequently, $\operatorname{Re}\left(e^{\mathrm{i} t} T\right) \phi=0$. Assumption e) now implies that $\langle\operatorname{Im} T \phi, \phi\rangle<0$ if $\phi \neq 0$. However, we showed, in (24), that $\langle-\operatorname{Im} T \phi, \phi\rangle=0$, that is, $\phi=0$ and therefore $T_{\sharp}$ is injective.

Hence, by assumption d) or e), $T_{\sharp}$ is an injective Fredholm operator on index 0 (Fredholmness is due to assumption b)) and hence boundedly invertible. By (22) we obtain

$$
\left\langle T_{\sharp} \psi, \psi\right\rangle \geq \frac{1}{\left\|T_{\sharp}\right\|}\left\|T_{\sharp} \psi\right\|^{2} \geq C\|\psi\|^{2} \quad \text { for all } \psi \in U
$$

Now, as $T_{\sharp}$ has been show to be positive definite, the square root $T_{\sharp}^{1 / 2}$ of $T_{\sharp}$ is also positive definite on $U$, see, e.g., [27], hence the inverse $T_{\sharp}^{-1 / 2}$ is bounded and we can write

$$
F_{\sharp}=F_{\sharp}^{1 / 2}\left(F_{\sharp}^{1 / 2}\right)^{*}=H^{*} T_{\sharp} H=\left(H^{*} T_{\sharp}^{1 / 2}\right)\left(H^{*} T_{\sharp}^{1 / 2}\right)^{*}
$$

However, if two positive operators agree, then the ranges of their square root agree, as the following well known lemma shows.

Lemma 4.2. (Lemma 2.4 in [19]). Let $V, U_{1}$ and $U_{2}$ be Hilbert spaces and $A_{j}: U_{j} \rightarrow V, j=1,2$, bounded and injective such that $A_{1} A_{1}^{*}=A_{2} A_{2}^{*}$. Then the ranges of $A_{1}$ and $A_{2}$ coincide and $A_{1}^{-1} A_{2}$ is an isomorphism from $U_{2}$ onto $U_{1}$.

Setting $A_{1}=F_{\sharp}^{1 / 2}$ and $A_{2}=H^{*} T_{\sharp}^{1 / 2}$, the last lemma states that the ranges of $F_{\sharp}^{1 / 2}$ and $H^{*} T_{\sharp}^{1 / 2}$ agree and that $F_{\sharp}^{-1 / 2} H^{*} T_{\sharp}^{1 / 2}$ is an isomorphism from $U$ to $V$. Since $T_{\sharp}^{1 / 2}$ is an isomorphism on $U$, we conclude that the range of $H^{*} T_{\sharp}^{1 / 2}$ equals the range of $H^{*}$ and that $F_{\sharp}^{-1 / 2} H^{*}: U \rightarrow V$ is bounded with bounded inverse.

\section{Study of the Middle Operator}

In this section we analyze the middle operator $T$ in the factorization of Theorem 3.2 and derive its necessary properties for the application of the Theorem 4.1. This is seen in the following lemma.

Lemma 5.1. Suppose that the contrast $q$ satisfies the Assumption 2.1 and that the direct scattering problem (9) is uniquely solvable for any $f \in L^{2}(D)^{3}$. Let $T: L^{2}(D)^{3} \rightarrow L^{2}(D)^{3}$ be the operator defined as in Theorem 3.2, i.e.

$$
T f=\operatorname{sign}(q)(f+\sqrt{|q|} \operatorname{curl} v),
$$

where $v \in H_{\alpha, l o c}(\operatorname{curl}, \Omega)$ is the radiating variational solution to

$$
\operatorname{curl}\left(\varepsilon_{\mathrm{r}}^{-1} \operatorname{curl} u\right)-k^{2} u=-\operatorname{curl}(q / \sqrt{|q|} f) .
$$

Then we have

(a) $T$ is injective and $\langle\operatorname{Im} T f, f\rangle \leq 0$ for all $f \in L^{2}(D)^{3}$. 
(b) Define the operator $T_{0}: L^{2}(D)^{3} \rightarrow L^{2}(D)^{3}$ by $T_{0} f=\operatorname{sign}(q)(f+\sqrt{|q|} \operatorname{curl} \tilde{v})$ where $\tilde{v} \in H_{\alpha, \text { loc }}(\operatorname{curl}, \Omega)$ solves (25) for $k=\mathrm{i}, f \in L^{2}(D)^{3}$, in the variational sense. Then we have that $T-T_{0}$ is compact in $L^{2}(D)^{3}$.

(c) For $T_{0}$ defined as in (b), if $\operatorname{Re}(q)>0$ on $L^{2}(D)^{3}$ then $\operatorname{Re}\left(T_{0}\right)$ is coercive in $L^{2}(D)^{3}$, i.e, there exists a constant $\gamma>0$ such that

$$
\left\langle\operatorname{Re}\left(T_{0}\right) f, f\right\rangle_{L^{2}(D)^{3}} \geq \gamma\|f\|_{L^{2}(D)^{3}} .
$$

Note that the proofs of (b) and (c) can be found in Theorem 4.9 [28] or Theorem 5.12 [20]. Here, for convenience, we repeat the proof of (b) in [20] with slight adaptations.

Proof. (a) We show the injectivity of $T$ by assuming that $T f=\operatorname{sign}(q)(f+\sqrt{|q|} \operatorname{curl} v)=0$, then $v$ is a radiating variational solution to the homogeneous problem $\operatorname{curl}^{2} v-k^{2} v=0$. However, we showed in the proof of Lemma 3.1 that the latter problem has only the trivial solution which implies that $v=0$ in $\Omega$. Thus, $f=0$ or $T$ is injective.

Now we set $w=f+\sqrt{|q|} \operatorname{curl} v$, then $T f=\operatorname{sign}(q) w$ and

$$
\begin{aligned}
\langle T f, f\rangle_{L^{2}(D)^{3}} & =\int_{D} \operatorname{sign}(q) w \cdot(\bar{w}-\sqrt{|q|} \operatorname{curl} \bar{v}) \mathrm{d} x \\
& =\int_{D}\left(\operatorname{sign}(q)|w|^{2}-q / \sqrt{|q|} w \cdot \operatorname{curl} \bar{v}\right) \mathrm{d} x
\end{aligned}
$$

For $r>\sup \left\{\left|x_{3}\right|:\left(x_{1}, x_{2}, x_{3}\right)^{\top} \in D\right\}$, we consider a smooth function $\chi \in C^{\infty}(\mathbb{R})$ such that $\chi=1$ in $\Omega_{r}$, $\chi=0$ in $\Omega \backslash \Omega_{2 r}$. Then $\chi v$ belongs to $H_{\alpha, \text { loc }}(\operatorname{curl}, \Omega)$ with compact support in $\Omega_{3 r}$. Since $v \in H_{\alpha, \text { loc }}(\operatorname{curl}, \Omega)$ is the radiating solution to $(25)$, we have

$$
\begin{aligned}
-\int_{D} q / \sqrt{|q|} w \cdot \operatorname{curl} \bar{v} \mathrm{~d} x & =\int_{\Omega_{r}}\left(|\operatorname{curl} v|^{2}-k^{2}|v|^{2}\right) \mathrm{d} x \\
& +\int_{\Omega_{2 r} \backslash \Omega_{r}}\left(\operatorname{curl} v \cdot \operatorname{curl}(\overline{\chi v})-k^{2} v \cdot \overline{\chi v}\right) \mathrm{d} x
\end{aligned}
$$

Now using Green's theorems and exploiting the fact that $v$ solve the Helmholtz equation in $\Omega \backslash \Omega_{h}$, we obtain that

$$
\begin{array}{r}
-\int_{D} q / \sqrt{|q|} w \cdot \operatorname{curl} \bar{v} \mathrm{~d} x=\int_{\Omega_{r}}\left(|\operatorname{curl} v|^{2}-k^{2}|v|^{2}\right) \mathrm{d} x+\left(\int_{\Gamma_{r}}-\int_{\Gamma_{-r}}\right)\left(e_{3} \times \operatorname{curl} v \cdot \bar{v}\right) \mathrm{d} s \\
=\int_{\Omega_{r}}\left(|\operatorname{curl} v|^{2}-k^{2}|v|^{2}\right) \mathrm{d} x+\left(\int_{\Gamma_{r}}-\int_{\Gamma_{-r}}\right)\left(-\overline{v_{1}} \partial_{3} v_{1}-\overline{v_{2}} \partial_{3} v_{2}+v_{3} \partial_{3} \overline{v_{3}}\right) \mathrm{d} s .
\end{array}
$$

Taking the imaginary part of the latter equation we have

$$
-\operatorname{Im} \int_{D} q / \sqrt{|q|} w \cdot \operatorname{curl} \bar{v} \mathrm{~d} x=\operatorname{Im}\left(\int_{\Gamma_{r}}-\int_{\Gamma_{-r}}\right)\left(-\overline{v_{1}} \partial_{3} v_{1}-\overline{v_{2}} \partial_{3} v_{2}+v_{3} \partial_{3} \overline{v_{3}}\right) \mathrm{d} s .
$$

Recall that $v$ satisfies the radiating Rayleigh condition for $\left|x_{3}\right|>r$. Thus all the terms corresponding to evanescent modes tend to zero as $r$ tends to infinity. Then due to a straightforward computation we derive

$$
\begin{aligned}
-\operatorname{Im} \int_{D} q / \sqrt{|q|} w \cdot \operatorname{curl} \bar{v} \mathrm{~d} x & =\lim _{r \rightarrow \infty} \operatorname{Im}\left(\int_{\Gamma_{r}}-\int_{\Gamma_{-r}}\right)\left(-\overline{v_{1}} \partial_{3} v_{1}-\overline{v_{2}} \partial_{3} v_{2}+v_{3} \partial_{3} \overline{v_{3}}\right) \mathrm{d} s \\
& =-4 \pi^{2} \sum_{j: k^{2}>\alpha_{j}^{2}} \beta_{j}\left(\left|\hat{v}_{j}^{+}\right|^{2}+\left|\hat{v}_{j}^{-}\right|^{2}\right),
\end{aligned}
$$


which implies that

$$
\begin{aligned}
\langle\operatorname{Im} T f, f\rangle_{L^{2}(D)^{3}} & =\int_{D} \operatorname{Im} q /|q||w|^{2} \mathrm{~d} x-\operatorname{Im} \int_{D} q / \sqrt{|q|} w \cdot \operatorname{curl} \bar{v} \mathrm{~d} x \\
& =\int_{D} \operatorname{Im} q /|q||w|^{2} \mathrm{~d} x-4 \pi^{2} \sum_{j: k^{2}>\alpha_{j}^{2}} \beta_{j}\left(\left|\hat{v}_{j}^{+}\right|^{2}+\left|\hat{v}_{j}^{-}\right|^{2}\right) \leq 0,
\end{aligned}
$$

since $\operatorname{Im}(q) \leq 0$ in $D$.

(b) From the definitions of $T$ and $T_{0}$ we note that $T f-T_{0} f=q / \sqrt{|q|} \operatorname{curl}(v-\tilde{v})$ where $v, \tilde{v} \in$ $H_{\alpha, \text { loc }}(\operatorname{curl}, \Omega)$ are the radiating solutions, for $k$ and $k=\mathrm{i}$, of

$$
\begin{array}{r}
\int_{\Omega}\left(\varepsilon_{\mathrm{r}}^{-1} \operatorname{curl} v \cdot \operatorname{curl} \bar{\psi}-k^{2} v \cdot \bar{\psi}\right) \mathrm{d} x=-\int_{\Omega} q / \sqrt{|q|} f \cdot \operatorname{curl} \bar{\psi} \mathrm{d} x, \\
\int_{\Omega}\left(\varepsilon_{\mathrm{r}}^{-1} \operatorname{curl} \tilde{v} \cdot \operatorname{curl} \bar{\psi}+\tilde{v} \cdot \bar{\psi}\right) \mathrm{d} x=-\int_{\Omega} q / \sqrt{|q|} f \cdot \operatorname{curl} \bar{\psi} \mathrm{d} x,
\end{array}
$$

respectively, for all $\psi \in H_{\alpha}(\operatorname{curl}, \Omega)$ with compact support. By substituting $\psi=\nabla \varphi$ for scalar functions $\varphi \in C^{\infty}(\Omega)$ with compact support we obtain that $\int_{\Omega} v \cdot \nabla \bar{\varphi} \mathrm{d} x=0$ for all $\varphi \in C^{\infty}(\Omega)$ with compact support which means that $\operatorname{div} v=0$, and analogously, $\operatorname{div} \tilde{v}=0$ in $\Omega$. The difference $w=v-\tilde{v}$ solves

$$
\int_{\Omega}\left(\varepsilon_{\mathrm{r}}^{-1} \operatorname{curl} w \cdot \operatorname{curl} \bar{\psi}-k^{2} w \cdot \bar{\psi}\right) \mathrm{d} x=\left(k^{2}+1\right) \int_{\Omega} \tilde{v} \cdot \bar{\psi} \mathrm{d} x
$$

for all $\psi \in H_{\alpha}(\operatorname{curl}, \Omega)$ with compact support.

Let now the sequence $f_{j}$ converge weakly to zero in $L^{2}(D)^{3}$ and denote by $v_{j}, \tilde{v}_{j} \in H_{\alpha, \text { loc }}(\operatorname{curl}, \Omega)$ the corresponding radiating solutions of (27) and (28), respectively. Define $w_{j} \in H_{\alpha, \text { loc }}(\operatorname{curl}, \Omega)$ again by the difference $w_{j}=v_{j}-\tilde{v}_{j}$. Set $R>\operatorname{supp}\left\{\left|x_{3}\right|:\left(x_{1}, x_{2}, x_{3}\right)^{\top} \in D\right\}$, then $\bar{D} \subset \Omega_{R}$. By the boundedness of the solution operator we conclude that $v_{j}$ and $\tilde{v}_{j}$ converge weakly to zero in $H_{\alpha}\left(\operatorname{curl}, \Omega_{R}\right)$. Furthermore, $v_{j}$ and $\tilde{v}_{j}$ are smooth outside of $\bar{D}$ and converges uniformly (with all of its derivatives) to zero on $\Gamma_{ \pm h}$. In consequence, $w_{j}$ converges to zero in $C\left(\partial \Omega_{R}\right)$. We determine $p_{j} \in H_{\alpha, \diamond}^{1}\left(\Omega_{R}\right)$ as the solution of

$$
\int_{\Omega_{R}} \nabla p_{j} \cdot \nabla \varphi \mathrm{d} x=\int_{\partial \Omega_{R}}\left(\nu \cdot w_{j}\right) \bar{\varphi} \mathrm{d} s
$$

for all $\varphi \in H_{\alpha, \diamond}^{1}\left(\Omega_{R}\right)$. Here the subspace $H_{\alpha, \diamond}^{1}\left(\Omega_{R}\right)$ of $H_{\alpha}^{1}\left(\Omega_{R}\right)$ is defined as $H_{\alpha, \diamond}^{1}\left(\Omega_{R}\right)=\left\{\varphi \in H_{\alpha}^{1}\left(\Omega_{R}\right)\right.$ : $\left.\int_{\Omega_{R}} \varphi \mathrm{d} s=0\right\}$. The solution of (29) exists and is unique since the form $(p, \varphi) \mapsto \int_{\Omega_{R}} \nabla p \cdot \nabla \varphi \mathrm{d} x$ is bounded and coercive on $H_{\alpha, \diamond}^{1}\left(\Omega_{R}\right)$ by the inequality of Poincaré (cf. [30]). The latter states that there exists a constant $c>0$ with

$$
\int_{\Omega_{R}}|\nabla \varphi|^{2} \mathrm{~d} x \geq c\|\varphi\|_{H_{\alpha}^{1}\left(\Omega_{R}\right)}^{2} \text { for all } \varphi \in H_{\alpha, \diamond}^{1}\left(\Omega_{R}\right) .
$$

Problem (29) is the variational form of the Neumann boundary value problem

$$
\Delta p_{j}=\operatorname{div} w_{j}=0 \text { in } \Omega_{R}, \quad \partial_{\nu} p_{j}=\nu \cdot w_{j} \text { on } \partial \Omega_{R} .
$$

We observe that (29) holds even for all $\varphi \in H_{\alpha}^{1}\left(\Omega_{R}\right)$ since $\int_{\partial \Omega_{R}}\left(\nu \cdot w_{j}\right) \mathrm{d} s$ vanishes by the divergence theorem and the fact that $\operatorname{div} w_{j}=0$. Substituting $\varphi=p_{j}$ into (29) yields, using (30) and the trace theorem,

$$
c\left\|p_{j}\right\|_{H_{\alpha}^{1}\left(\Omega_{R}\right)}^{2} \leq \int_{\Omega_{R}}\left|\nabla p_{j}\right|^{2} \mathrm{~d} x=\int_{\partial \Omega_{R}}\left(\nu \cdot w_{j}\right) \overline{p_{j}} \mathrm{~d} s \leq \tilde{c}\left\|w_{j}\right\|_{C\left(\partial \Omega_{R}\right)}\left\|p_{j}\right\|_{H_{\alpha}^{1}\left(\Omega_{R}\right)},
$$

i.e. $\left\|p_{j}\right\|_{H_{\alpha}^{1}\left(\Omega_{R}\right)} \leq(\tilde{c} / c)\left\|w_{j}\right\|_{C\left(\partial \Omega_{R}\right)}$ which converges to zero. Therefore, the functions $\tilde{w}_{j}:=w_{j}-\nabla p_{j} \in$ $H_{\alpha}\left(\operatorname{curl}, \Omega_{R}\right)$ satisfy 
- $\tilde{w}_{j} \in H_{\alpha, \mathrm{div}}\left(\operatorname{curl}, \Omega_{R}\right):=\left\{u \in H_{\alpha}\left(\operatorname{curl}, \Omega_{R}\right): \int_{\Omega_{R}} \nabla \varphi \cdot u \mathrm{~d} x=0\right.$ for all $\left.\varphi \in H_{\alpha}^{1}\left(\Omega_{R}\right)\right\}$

- $\tilde{w}_{j} \rightarrow 0$ weakly in $L^{2}\left(\Omega_{R}\right)^{3}$,

- $\operatorname{curl} \tilde{w}_{j}=\operatorname{curl} w_{j} \rightarrow 0$ weakly in $L^{2}\left(\Omega_{R}\right)^{3}$.

These three conditions assure that $\tilde{w}_{j}$ converges to zero in the norm of $L^{2}\left(\Omega_{R}\right)^{3}$ since the closed subspace $H_{\alpha, \text { div }}\left(\operatorname{curl}, \Omega_{R}\right)$ of $H_{\alpha}\left(\operatorname{curl}, \Omega_{R}\right)$ is compactly embedded in $L^{2}(\Omega)^{3}$. We refer to [31], see also [26], Theorem 4.7. Since also $\left\|\nabla p_{j}\right\|_{L^{2}\left(\Omega_{R}\right)^{3}} \rightarrow 0$ this yields $\left\|w_{j}\right\|_{L^{2}\left(\Omega_{R}\right)} \rightarrow 0$ as $j$ tends to infinity. Now we return to the variational equation for $w_{j}$ and substitute $\psi=\phi \overline{w_{j}}$ where $\phi \in C^{\infty}(\Omega)$ is some function with compact support such that $\phi=1$ on $\Omega_{R}$. This yields

$$
\begin{aligned}
\int_{\Omega_{R}}\left(\varepsilon_{\mathrm{r}}^{-1}\left|\operatorname{curl} w_{j}\right|^{2}-k^{2}\left|w_{j}\right|^{2}\right) \mathrm{d} x & =\int_{\Omega \backslash \Omega_{R}}\left(\varepsilon_{\mathrm{r}}^{-1} \operatorname{curl} w_{j} \cdot \operatorname{curl}\left(\phi \overline{w_{j}}\right)-k^{2} \phi\left|w_{j}\right|^{2}\right) \mathrm{d} x \\
& +\left(k^{2}+1\right) \int_{\Omega} \phi \tilde{v_{j}} \cdot \overline{w_{j}} \mathrm{~d} x .
\end{aligned}
$$

We note that $w_{j}$ is smooth in $\Omega \backslash \Omega_{R}$. Green's theorem in $\Omega_{m R} \backslash \Omega_{R}$ (for a sufficiently large value of $m$ ) and application of $\operatorname{curl}^{2} w_{j}-k^{2} w_{j}=\left(k^{2}+1\right) \tilde{v}_{j}$ in this region yields

$$
\int_{\Omega_{R}}\left(\varepsilon_{\mathrm{r}}^{-1}\left|\operatorname{curl} w_{j}\right|^{2}-k^{2}\left|w_{j}\right|^{2}\right) \mathrm{d} x=\int_{\partial \Omega_{R}}\left(\nu \times \operatorname{curl} w_{j}\right) \cdot \overline{w_{j}} \mathrm{~d} s+\left(k^{2}+1\right) \int_{\Omega_{R}}{\tilde{v_{j}}}_{\overline{w_{j}}} \mathrm{~d} x
$$

which tends to zero as $j$ tends to infinity since $\tilde{v}_{j}$ and $\operatorname{curl} w_{j}$ are bounded sequences and $\left\|w_{j}\right\|_{L^{2}\left(\Omega_{R}\right)}$, $\left\|w_{j}\right\|_{C\left(\partial \Omega_{R}\right)}$ tend to zero. Therefore, also curl $w_{j}$ tends to zeros in $L^{2}\left(\Omega_{R}\right)^{3}$ which complete the proof.

\section{Characterization of the Biperiodic Support}

In this section, we give a characterization for a point $z$ belonging to the support of the contrast $q$ by exploiting special test sequences. A simple criterion for imaging the periodic support is also proposed.

First we introduce some basic facts about $\alpha$-quasiperiodic Green functions. It is well known that the function $G_{k}(x, y)$ given by

$$
G_{k}(x, y)=\frac{\mathrm{i}}{8 \pi^{2}} \sum_{j \in \mathbb{Z}} \frac{1}{\beta_{j}} e^{\mathrm{i} \alpha_{j} \cdot(x-y)+\mathrm{i} \beta_{j}\left|x_{3}-y_{3}\right|}, \quad x, y \in \Omega, x_{3} \neq y_{3},
$$

is the $\alpha$-quasiperiodic Green's function of the Helmholtz operator in three dimensions. That means, for fixed $y \in \Omega$,

$$
\Delta_{x} G_{k}(x, y)+k^{2} G_{k}(x, y)=-\delta_{y}(x), \quad x \in \Omega .
$$

Also, another form of $G_{k}(x, y)$ can be given, see e.g. [2],

$$
G_{k}(x, y)=\frac{e^{\mathrm{i} k|x-y|}}{4 \pi|x-y|}+\Psi_{k}(x-y)
$$

where $\Psi_{k}$ is the analytic solution to the Helmholtz equation in $(-2 \pi, 2 \pi)^{2} \times \mathbb{R}$.

The $\alpha$-quasiperiodic Green's tensor $\mathbb{G}_{k}(x, y) \in \mathbb{C}^{3 \times 3}$ defined by

$$
\mathbb{G}_{k}(x, y)=G_{k}(x, y) \mathrm{I}_{3 \times 3}+k^{-2} \nabla_{x} \operatorname{div}{ }_{x}\left(G_{k}(x, y) \mathrm{I}_{3 \times 3}\right), \quad x, y \in \Omega, x_{3} \neq y_{3},
$$

solves

$$
\operatorname{curl}_{x}^{2} \mathbb{G}_{k}(x, y)-k^{2} \mathbb{G}_{k}(x, y)=\delta_{y}(x) \mathrm{I}_{3 \times 3}, \quad x \in \Omega,
$$

where $\mathrm{I}_{3 \times 3}$ is the identity matrix. Here, the curl of a matrix is taken columnwise, the div of a matrix and the $\nabla$ are meant to be taken columnwise and componentwise, respectively. Note that $\mathbb{G}_{k}$ satisfies the Rayleigh expansion condition and has a strong singularity due to the representation of $G_{k}$ in (32). 
Lemma 6.1. Let the operator $W$ be defined as in (18). For any $z \in \Omega$ and fixed nonzero $p=\left(p_{1}, p_{2}, p_{3}\right) \in \mathbb{C}^{3}$ we denote by $\left(\hat{\Psi}_{z, j}^{ \pm}\right)_{j \in \mathbb{Z}^{2}} \in \ell^{2}\left(\mathbb{Z}^{2}\right)^{4}$ the Rayleigh coefficients of the first two components of

$$
\begin{aligned}
\Psi_{z}(x) & :=k^{2} \mathbb{G}_{k}(x, z) p \\
& =\left(\begin{array}{c}
{\left[k^{2} G_{k}(x, z)+\frac{\partial^{2} G_{k}(x, z)}{\partial x_{1}^{2}}\right] p_{1}+\frac{\partial^{2} G_{k}(x, z)}{\partial x_{1} \partial x_{2}} p_{2}+\frac{\partial^{2} G_{k}(x, z)}{\partial x_{1} \partial x_{3}} p_{3}} \\
\frac{\partial^{2} G_{k}(x, z)}{\partial x_{2} x_{1}} p_{1}+\left[k^{2} G_{k}(x, z)+\frac{\partial^{2} G_{k}(x, z)}{\partial x_{2}^{2}}\right] p_{2}+\frac{\partial^{2} G_{k}(x, z)}{\partial x_{2} \partial x_{3}} p_{3} \\
\frac{\partial^{2} G_{k}(x, z)}{\partial x_{3} \partial x_{1}} p_{1}+\frac{\partial^{2} G_{k}(x, z)}{\partial x_{3} \partial x_{2}} p_{2}+\left[k^{2} G_{k}(x, z)+\frac{\partial^{2} G_{k}(x, z)}{\partial x_{3}^{2}}\right] p_{3}
\end{array}\right),
\end{aligned}
$$

for $x \in \Omega, x \neq z$. Then $z$ belongs to $D$ if and only if $W\left(\hat{\Psi}_{j, z}^{ \pm}\right) \in \operatorname{Rg}\left(H^{*}\right)$.

Remark 6.2. Note that the Rayleigh sequences $\hat{G}_{k, j}^{ \pm}(z)$ of the $\alpha$-quasiperiodic Green's function $G_{k}(\cdot, z)$ can be obtained from the representation of $G_{k}(\cdot, z)$ in (31)

$$
\hat{G}_{k, j}^{ \pm}(z)=\frac{\mathrm{i}}{8 \pi^{2} \beta_{j}} e^{-\mathrm{i}\left[\alpha_{1, j} z_{1}+\alpha_{2, j} z_{2} \pm \beta_{j}\left(z_{3} \mp h\right)\right]} .
$$

Then the Rayleigh sequences $\left(\hat{\Psi}_{z, j}^{ \pm}\right)_{j \in \mathbb{Z}^{2}} \in \ell^{2}\left(\mathbb{Z}^{2}\right)^{4}$ of the first two components of $\Psi_{z}$ can be given as

$$
\hat{\Psi}_{z, j}^{ \pm}=\left(\begin{array}{c}
\left(k^{2}-\alpha_{1, j}^{2}\right) \hat{G}_{k, j}^{ \pm}(z) p_{1}-\alpha_{1, j} \alpha_{2, j} \hat{G}_{k, j}^{ \pm}(z) p_{2} \mp \alpha_{1, j} \beta_{j} \hat{G}_{k, j}^{ \pm}(z) p_{3} \\
-\alpha_{2, j} \alpha_{1, j} \hat{G}_{k, j}^{ \pm}(z) p_{1}+\left(k^{2}-\alpha_{2, j}^{2}\right) \hat{G}_{k, j}^{ \pm}(z) p_{2} \mp \alpha_{2, j} \beta_{j} \hat{G}_{k, j}^{ \pm}(z) p_{3}
\end{array}\right) .
$$

Proof. First, let $z \in D$. Recall the operator $Q$ defined in (19). Due to the fact that $H^{*}=-W Q$, it is sufficient to show that $\left(\hat{\Psi}_{z, j}\right)_{j \in \mathbb{Z}^{2}} \in \operatorname{Rg}(Q)$. Choose $r>0$ such that $B(z, r) \in D$ and consider a cut-off function $\varphi \in C^{\infty}\left(\mathbb{R}^{3}\right)$ with $\varphi(x)=0$ for $|x-z| \leq r / 2$ and $\varphi(x)=1$ for $|x-z| \geq r$. We define

$$
w(x)=\operatorname{curl}^{2}\left(\varphi(x) \mathbb{G}_{k}(x, z) p\right), \quad x \in \Omega .
$$

Note that, for $|x-z| \geq r$, we have

$$
w(x)=\operatorname{curl}^{2}\left(\varphi(x) \mathbb{G}_{k}(x, z) p\right)=k^{2} \mathbb{G}_{k}(x, z) p,
$$

and further $\left(\hat{w}_{j}\right)_{j \in \mathbb{Z}^{2}}=\left(\hat{\Psi}_{z, j}\right)_{j \in \mathbb{Z}^{2}}$. Using Green's theorem we obtain

$$
\begin{aligned}
\int_{\Omega}\left(\operatorname{curl} w \cdot \operatorname{curl} \bar{\psi}-k^{2} w \cdot \bar{\psi}\right) \mathrm{d} x & =\int_{\Omega}\left(\operatorname{curl} w-k^{2} \operatorname{curl}\left(\varphi(x) \mathbb{G}_{k}(x, z) p\right)\right) \cdot \operatorname{curl} \bar{\psi} \mathrm{d} x \\
& =\int_{\Omega} g \cdot \operatorname{curl} \bar{\psi} \mathrm{d} x,
\end{aligned}
$$

for all $\psi \in H_{\alpha}(\operatorname{curl}, \Omega)$ with compact support, and $g:=\operatorname{curl} w-k^{2} \operatorname{curl}\left(\varphi(x) \mathbb{G}_{k}(x, z) p\right)$. Since $g$ is smooth and vanishes for $|z-x| \geq r$, thus $\operatorname{supp}(g) \subset D$. Set $f=\sqrt{|q|}^{-1} g \in L^{2}(D)^{3}$. Then we have

$$
\int_{\Omega}\left(\operatorname{curl} w \cdot \operatorname{curl} \bar{\psi}-k^{2} w \cdot \bar{\psi}\right) \mathrm{d} x=\int_{D} \sqrt{|q|} f \cdot \operatorname{curl} \bar{\psi} \mathrm{d} x
$$

which implies that $\left(\hat{\Psi}_{z, j}\right)_{j \in \mathbb{Z}^{2}} \in \operatorname{Rg}(Q)$.

Now let $z \notin D$, and on the contrary, assume that $\hat{\Psi}_{z, j} \in \operatorname{Rg}(Q)$. That means there exists $u \in$ $H_{\alpha, \text { loc }}(\operatorname{curl}, \Omega)$ and $f \in L^{2}(D)^{3}$ such that $u$ is the variational radiating solution to $\operatorname{curl}^{2} u-k^{2} u=\operatorname{curl}(\sqrt{|q|} f)$ and $\hat{u}_{j}=\hat{\Psi}_{z, j}$ for all $j \in \mathbb{Z}^{2}$. Since the Rayleigh sequences of $u$ and $\Psi_{z}$ are equal, both functions coincide in $(0,2 \pi)^{2} \times\left\{\left|x_{3}\right|>h\right\}$ where $h>\operatorname{supp}\left\{\left|x_{3}\right|:\left(x_{1}, x_{2}, x_{3}\right)^{\top} \in D\right\}$. Due to the analyticity of $u$ and $\Psi_{z}$ in $\Omega \backslash D$ and $\Omega \backslash\{z\}$, respectively, and the analytic continuation we conclude that $u=\Psi_{z}$ in $\Omega \backslash(D \cup\{z\})$. This is a contradiction since $u \in H(\operatorname{curl}, B)$ for any ball $B$ containing $z$ but $\operatorname{curl}\left(k^{2} \mathbb{G}_{k}(\cdot, z) p\right) \notin H(\operatorname{curl}, B)$ due to a strongly singularity at $z$. 
Theorem 6.3. Suppose that the contrast $q$ satisfies the Assumption 2.1 and that the direct scattering problem (9) is uniquely solvable. For $j \in \mathbb{Z}^{2}$, denote by $\left(\lambda_{n}, \psi_{n, j}\right)_{n \in \mathbb{N}}$ the orthonormal eigensystem of $(W N)_{\sharp}=|\operatorname{Re}(W N)|+\operatorname{Im}(W N)$ and by $\left(\hat{\Psi}_{z, j}^{ \pm}\right)_{j \in \mathbb{Z}^{2}}$ the test sequence in Lemma 6.1. A point $z$ belongs to the support of $q$ if and only if

$$
\sum_{n=1}^{\infty} \frac{\left|\left\langle\hat{\Psi}_{z, j}^{ \pm}, \psi_{j, n}\right\rangle_{\ell^{2}\left(\mathbb{Z}^{2}\right)^{4}}\right|^{2}}{\lambda_{n}}<\infty .
$$

Proof. As we assumed in the theorem, $\left(\lambda_{n}, \psi_{n, j}\right)_{n \in \mathbb{N}}$ is an orthonormal eigensystem of $(W N)_{\sharp}$. The assumptions of Theorem 4.1 on $H, H^{*}$ and $T$ in the factorization $W N=H^{*} T H$ have been checked in Lemmas 3.1 and 5.1. Therefore, an application of Theorem 4.1 yields that $\operatorname{Rg}\left((W N)_{\sharp}^{1 / 2}\right)=\operatorname{Rg}\left(H^{*}\right)$. Combining this range identity with the characterization given in Lemma 6.1 we obtain that $\left(\hat{\Psi}_{z, j}^{ \pm}\right)_{j \in \mathbb{Z}^{2}} \in \operatorname{Rg}\left((W N)_{\sharp}^{1 / 2}\right)$ if and only if $z \in D$. Then the criterion (33) follows from the Picard's range criterion.

\section{Numerical Experiments}

As mentioned in the introduction, these are to the best of our knowledge the first three-dimensional examples of the method in a biperiodic setting. These numerical examples focus on the dependence of the reconstructions on the number of the incident fields (or, equivalently, the evanescent modes), and the performance of the method when the data is perturbed by artificial noise. Further, we also indicate the number of the evanescent and propagating modes which are used for each reconstruction. These experiments use three biperiodic structures presented in one period $\Omega=(-\pi, \pi)^{2} \times \mathbb{R}$ in terms of the support $\bar{D}$ of the contrast $q$ as follows:

(i) Biperiodic structures of ellipsoids,

$$
\begin{aligned}
\bar{D} & =\left\{\left(x_{1}, x_{2}, x_{3}\right)^{\top} \in \Omega: \frac{x_{1}^{2}}{2.5^{2}}+\frac{x_{2}^{2}}{2.5^{2}}+\frac{x_{3}^{2}}{0.4^{2}} \leq 1\right\}, \\
q & =0.5 \text { in } D .
\end{aligned}
$$

(ii) Biperiodic structures of cubes,

$$
\begin{aligned}
\bar{D} & =\left\{\left(x_{1}, x_{2}, x_{3}\right)^{\top} \in \Omega:\left|x_{1}\right| \leq 2.5,\left|x_{2}\right| \leq 2.5,\left|x_{3}\right| \leq 0.45\right\}, \\
q & =\left(x_{3}+1\right)\left(\sin \left(x_{1}\right)^{2} \sin \left(x_{2}\right)^{2}+0.3\right) / 4-0.4 \mathrm{i} \quad \text { in } D .
\end{aligned}
$$

(iii) Biperiodic structures of plus signs,

$$
\begin{aligned}
\bar{D} & =\Omega \cap\left[\left(\left\{\left|x_{1}\right| \leq 1.75\right\} \cup\left\{\left|x_{2}\right| \leq 1.75\right\}\right) \cap\left\{\left|x_{3}\right| \leq 0.45\right\}\right], \\
q & =\left\{\begin{array}{l}
0.5-0.6 \mathrm{i} \text { in } D_{1}=\left\{\left(x_{1}, x_{2}\right)^{\top} \in D:-1<x_{1}<1\right\}, \\
0.3 \text { in } D \backslash D_{1} .
\end{array}\right.
\end{aligned}
$$

The data of the direct scattering problem has been obtained by the extension of the volume integral equation method studied in [24] for the scalar case to the Maxwell's equations. Of course it is not possible to numerically compute data for all incident fields $\left(\varphi_{j}^{(l) \pm}\right)_{j \in \mathbb{Z}^{2}}$ in (11). Denote

$$
\mathbb{Z}_{M_{1}, M_{2}}^{2}=\left\{j=\left(j_{1}, j_{2}\right) \in \mathbb{Z}^{2}:-M_{1} \leq j_{1}, j_{2} \leq M_{2}\right\}, \quad M_{1}, M_{2} \in \mathbb{N} .
$$

For the numerical experiments here we solve the direct problem for a number $j=\left(j_{1}, j_{2}\right)$ of incident fields $\varphi_{j}^{(l) \pm}$ where $j \in \mathbb{Z}_{M 1, M 2}^{2}$. Denote by $\mathcal{N}_{M_{1}, M_{2}}$ the block matrix corresponding to the discretization of the near field operator $N$. Then $\mathcal{N}_{M_{1}, M_{2}}$ is given by

$$
\mathcal{N}_{M_{1}, M_{2}}=\left(\begin{array}{cccc}
\left(\hat{u}_{1, n}^{+}\right)_{j}^{(1)+} & \left(\hat{u}_{1, n}^{+}\right)_{j}^{(1)-} & \left(\hat{u}_{1, n}^{+}\right)_{j}^{(2)+} & \left(\hat{u}_{1, n}^{+}\right)_{j}^{(2)-} \\
\left(\hat{u}_{2, n}^{+}\right)_{j}^{(1)+} & \left(\hat{u}_{2, n}^{+}\right)_{j}^{(1)-} & \left(\hat{u}_{2, n}^{+}\right)_{j}^{(2)+} & \left(\hat{u}_{2, n}^{+}\right)_{j}^{(2)-} \\
\left(\hat{u}_{1, n}^{-}\right)_{j}^{(1)+} & \left(\hat{u}_{1, n}^{-}\right)_{j}^{(1)-} & \left(\hat{u}_{1, n}^{-}\right)_{j}^{(2)+} & \left(\hat{u}_{1, n}^{-}\right)_{j}^{(2)-} \\
\left(\hat{u}_{2, n}^{-}\right)_{j}^{(1)+} & \left(\hat{u}_{2, n}^{-}\right)_{j}^{(1)-} & \left(\hat{u}_{2, n}^{-}\right)_{j}^{(2)+} & \left(\hat{u}_{2, n}^{-}\right)_{j}^{(2)-}
\end{array}\right), \quad j, n \in \mathbb{Z}_{M_{1}, M_{2}}^{2} .
$$


Here $\hat{u}_{(1,2), n}^{ \pm}$are the Rayleigh sequences defined in (10) while for $l=1,2,(\cdot)_{j}^{(l) \pm}$ indicate the correspondence to the the incident fields $\varphi_{j}^{(l) \pm}$. Note that each component of $\mathcal{N}_{M_{1}, M_{2}}$ is a matrix of size $\left(M_{1}+M_{2}+1\right)^{2}$, thus $\mathcal{N}_{M_{1}, M_{2}}$ is a $4\left(M_{1}+M_{2}+1\right)^{2} \times 4\left(M_{1}+M_{2}+1\right)^{2}$ matrix. The matrix $\mathcal{W N}_{M_{1}, M_{2}}$ which corresponds to the discretization of $W N$ can be computed using (16), the symmetric matrix $\operatorname{Re}\left(\mathcal{W N}_{M_{1}, M_{2}}\right)$ can be decomposed as

$$
\operatorname{Re}\left(\mathcal{W} \mathcal{N}_{M_{1}, M_{2}}\right)=V D V^{-1},
$$

where $D, V$ are the matrices of eigenvalues and corresponding eigenvectors of $\operatorname{Re}\left(\mathcal{W N}_{M_{1}, M_{2}}\right)$, respectively. Denote by $|D|$ the absolute value of $D$ which is taken componentwise. Then we have

$$
\left(\mathcal{W N}_{M_{1}, M_{2}}\right)_{\sharp}:=V|D| V^{-1}+\operatorname{Im}\left(\mathcal{W} \mathcal{N}_{M_{1}, M_{2}}\right) .
$$

Computing singular value decomposition of $\left(\mathcal{W N}_{M_{1}, M_{2}}\right)_{\sharp}$ implies that

$$
\left(\mathcal{W} \mathcal{N}_{M_{1}, M_{2}}\right)_{\sharp}^{1 / 2}=U|S|^{1 / 2} V^{-1},
$$

where $S$ is the diagonal matrix of singular values $\lambda_{m}$ of $\left(\mathcal{W N}_{M_{1}, M_{2}}\right)_{\sharp}$. Also $U=\left[\psi_{n, m}\right]$ is a $4\left(M_{1}+M_{2}+\right.$ $1)^{2} \times 4\left(M_{1}+M_{2}+1\right)^{2}$ matrix of "left" singular vectors. We now reshape $\left[\psi_{n, m}\right]$ into 4 arrays $\left[\psi_{j+M_{1}+1, m}^{(l)}\right]$, $l=1, \ldots, 4, j \in \mathbb{Z}_{M_{1}, M_{2}}^{2}$, where each of them consists of $4\left(M_{1}+M_{2}+1\right)^{2}$ square matrices of size $M_{1}+M_{2}+1$. Note that the elements of $\left[\psi_{j+M_{1}+1, m}^{(l)}\right]$ are taken columnwise from $\left[\psi_{n, m}\right]$.

Now recall that we have $\hat{\Psi}_{z, j}^{ \pm}=\left(\hat{\Psi}_{1, z, j}^{+}, \hat{\Psi}_{2, z, j}^{+}, \hat{\Psi}_{1, z, j}^{-}, \hat{\Psi}_{2, z, j}^{-}\right)^{\top}$ which can be rewritten as

$$
\hat{\Psi}_{z, j}=\left(\hat{\Psi}_{z, j}^{(1)}, \hat{\Psi}_{z, j}^{(2)}, \hat{\Psi}_{z, j}^{(3)}, \hat{\Psi}_{z, j}^{(4)}\right)^{\top}
$$

Then the criterion (33) for computing the image can be approximated as follows

$$
P(z)=\left[\sum_{n=1}^{4\left(M_{1}+M_{2}+1\right)^{2}} \frac{A_{n}(z)}{\lambda_{n}}\right]^{-1},
$$

where

$$
A_{n}(z)=\left|\sum_{l=1}^{4} \sum_{j \in \mathbb{Z}_{M_{1}, M_{2}}^{2}} \hat{\Psi}_{z, j}^{(l)} \bar{\psi}_{j+M_{1}+1, n}^{(l)}\right|^{2} .
$$

Note that $P$ should be small outside of $D$ and big inside of $D$.

To show the performance of the method with noisy data, we pertub our synthetic data by artificial noise. More particularly, we add the noise matrix $\mathcal{X}$ of uniformly distributed random entries to the data matrix $\left(\mathcal{W N}_{M_{1}, M_{2}}\right)_{\sharp}^{1 / 2}$. Denote by $\delta$ the noise level, then the noise data matrix $\left(\mathcal{W} \mathcal{N}_{M_{1}, M_{2}}\right)_{\sharp, \delta}^{1 / 2}$ is given by

$$
\left(\mathcal{W} \mathcal{N}_{M_{1}, M_{2}}\right)_{\sharp, \delta}^{1 / 2}:=\left(\mathcal{W N}_{M_{1}, M_{2}}\right)_{\sharp}^{1 / 2}+\delta \frac{\mathcal{X}}{\|\mathcal{X}\|_{2}}\left\|\left(\mathcal{W N}_{M_{1}, M_{2}}\right)_{\sharp}^{1 / 2}\right\|_{2},
$$

where $\|\cdot\|_{2}$ is the matrix 2-norm. Note that from the latter equation we also have

$$
\frac{\left\|\left(\mathcal{W N}_{M_{1}, M_{2}}\right)_{\sharp, \delta}^{1 / 2}-\left(\mathcal{W} \mathcal{N}_{M_{1}, M_{2}}\right)_{\sharp}^{1 / 2}\right\|_{2}}{\left\|\left(\mathcal{W} \mathcal{N}_{M_{1}, M_{2}}\right)_{\sharp}^{1 / 2}\right\|_{2}}=\delta .
$$

Since we apply Tikhonov regularization [12], instead of implementing (35) we consider

$$
P(z)=\left[\sum_{n=1}^{4\left(M_{1}+M_{2}+1\right)^{2}}\left(\frac{\lambda_{n}^{1 / 2}}{\lambda_{n}+\gamma}\right)^{2} A_{n}(z)\right]^{-1},
$$


where

$$
A_{n}(z)=\left|\sum_{l=1}^{4} \sum_{j \in \mathbb{Z}_{M_{1}, M_{2}}^{2}} \hat{\Psi}_{z, j}^{(l)} \bar{\psi}_{j+M_{1}+1, n}^{(l)}\right|^{2} .
$$

Here $\lambda_{n}, \psi_{j, n}$ are the singular values and vectors of $\left(\mathcal{W} \mathcal{N}_{M_{1}, M_{2}}\right)_{\sharp, \delta}$, respectively. The parameter $\gamma$ is chosen by Morozov's generalized discrepancy principle which can be obtained by solving the equation

$$
\sum_{n=1}^{4\left(M_{1}+M_{2}+1\right)^{2}} \frac{\gamma^{2}-\delta^{2} \lambda_{n}}{\left(\lambda_{n}+\gamma\right)^{2}} A_{n}(z)=0,
$$

for each sampling point $z$. For the following experiments, we choose the wave number $k=2 \pi / 3$. The number of the incident fields used is $4\left(M_{1}+M_{2}+1\right)^{2}$. Further, the reconstructions have been smoothened using the command smooth3 in Matlab, and we plot the pictures in $3 \times 3$ periods.

\section{References}

[1] H. Ammari, Uniqueness theorems for an inverse problem in a doubly periodic structures, Inverse Problems, 11 (1995), pp. 823-833.

[2] T. Arens, Scattering by biperiodic layered media: The integral equation approach. Habilitation Thesis, Universität Karlsruhe, 2010.

[3] T. Arens, Why linear sampling works, Inverse Problems, 20 (2004), pp. 163-173.

[4] T. Arens and N. Grinberg, A complete factorization method for scattering by periodic structures, Computing, 75 (2005), pp. 111-132.

[5] T. Arens And A. Kirsch, The factorization method in inverse scattering from periodic structures, Inverse Problems, 19 (2003), pp. 1195-1211.

[6] T. Arens And A. Lechleiter, The linear sampling method revisited, J. Int. Eq. Appl., 21 (2009), pp. $179-202$.

[7] G. BAO, Variational approximation of Maxwell's equations in biperiodic structures, SIAM J. Appl. Math., 57 (1997), pp. 364-381.

[8] G. BaO, H. Zhang, and J. Zou, Unique determination of periodic polyhedral structures scattered electromagnetic fields, Trans. Amer. Math. Soc, 9 (2011), pp. 4527-4551.

[9] G. BaO And Z. Zhou, An inverse problem for scattering by a doubly periodic structure, Trans. Amer. Math. Soc, 350 (1998), pp. 4089-4103.

[10] F. Cakoni and D. Colton, Qualitative Methods in Inverse Scattering Theory. An Introduction., Springer, Berlin, 2006.

[11] F. Cakoni, D. Colton, And P. Monk, The Linear Sampling Method in Inverse Electromagnetic Scattering, SIAM, 2011.

[12] D. Colton, J. Coyle, And P. Monk, Recent developments in inverse acoustic scattering theory, SIAM Review, 42 (2000), pp. 396-414.

[13] D. Colton And A. Kirsch, A simple method for solving inverse scattering problems in the resonance region, Inverse Problems, 12 (1996), pp. 383-393. 


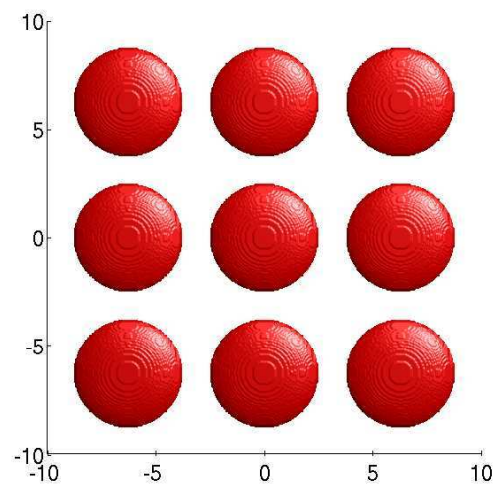

(a) Exact geometry (view down $x_{3}$ axis)

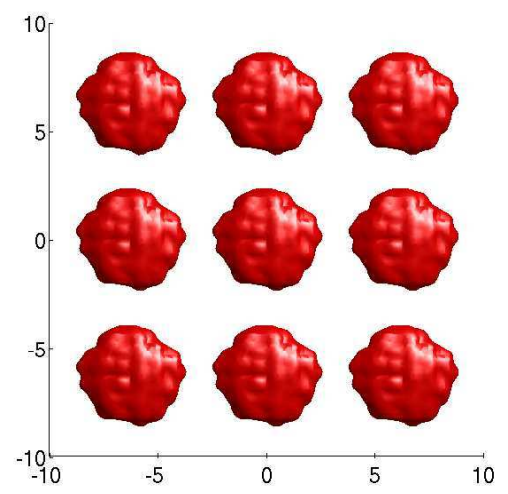

(c) $M_{1,2}=4$ (view down $x_{3}$ axis)

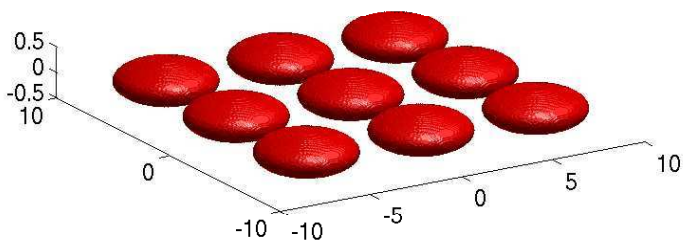

(e) Exact geometry (3D view)

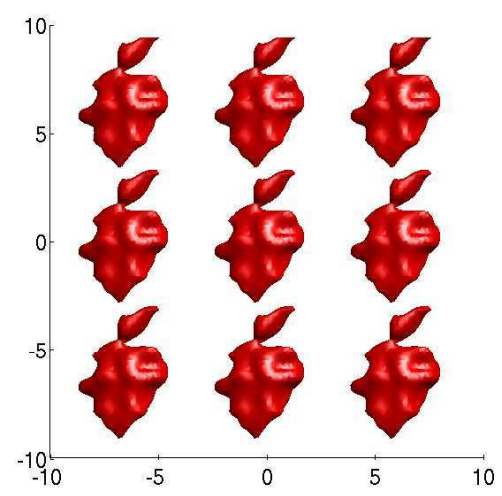

(b) $M_{1,2}=2$ (view down $x_{3}$ axis)

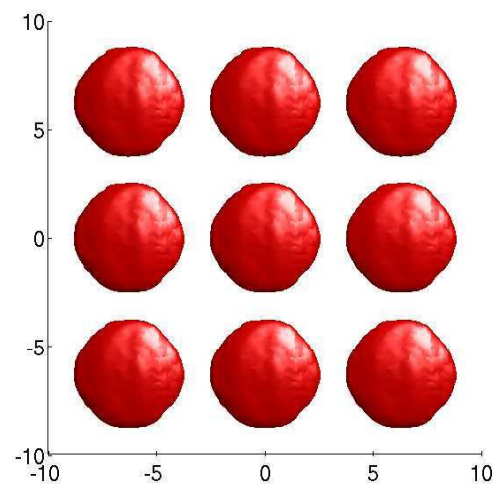

(d) $M_{1,2}=8$ (view down $x_{3}$ axis)

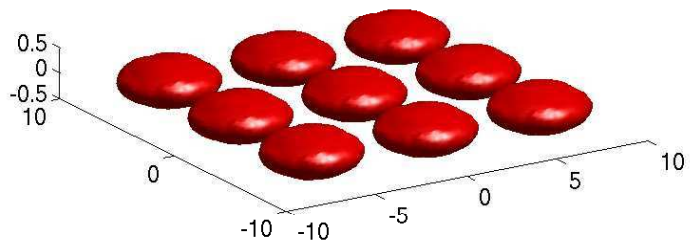

(f) $M_{1,2}=8(3 \mathrm{D}$ view $)$

Figure 1: Reconstructions of biperiodic shapes of ellipsoids for different number of incident fields without noise. The number of Rayleigh coefficients measured in each reconstruction is $4\left(M_{1}+M_{2}+1\right)^{2}$. The contrast $q=0.5$ in $D$. (b) 48 propagating modes, 52 evanescent modes, isovalue 7 (c) 52 propagating modes, 312 evanescent modes, isovalue 0.1 (d) 52 propagating modes, 1104 evanescent modes, isovalue 0.01 . 


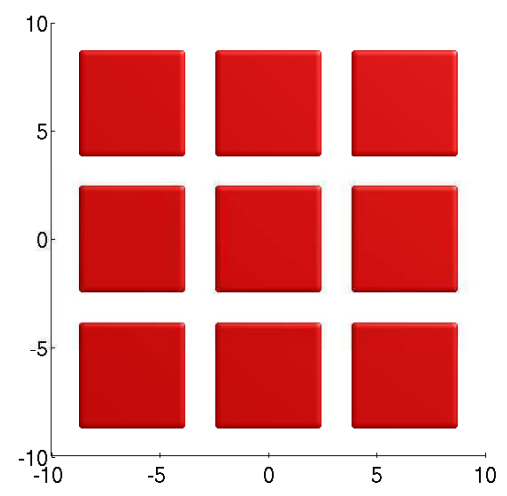

(a) Exact geometry (view down $x_{3}$ axis)

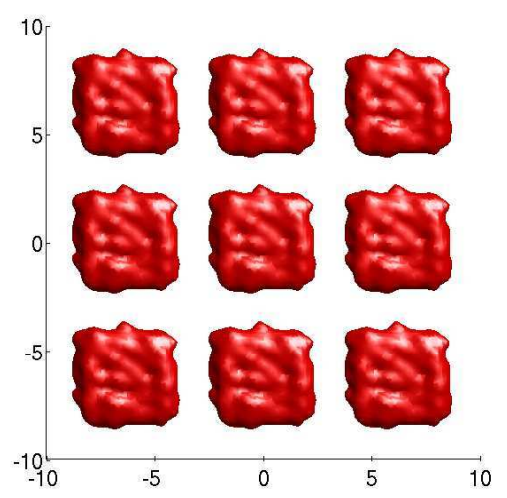

(c) $M_{1,2}=4$ (view down $x_{3}$ axis)

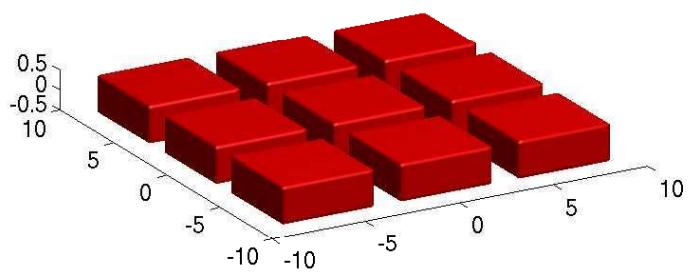

(e) Exact geometry (3D view)

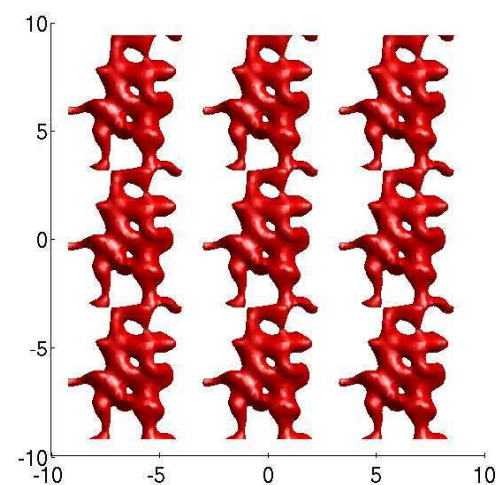

(b) $M_{1,2}=2$ (view down $x_{3}$ axis)

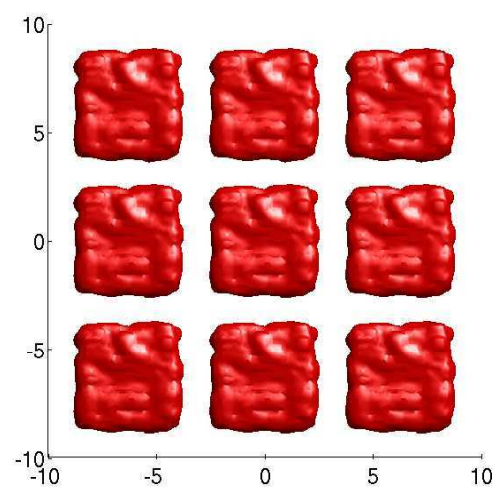

(d) $M_{1,2}=8$ (view down $x_{3}$ axis)

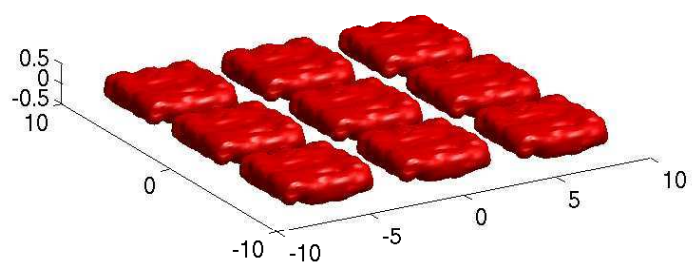

(f) $M_{1,2}=8$ (3D view)

Figure 2: Reconstructions of biperiodic shapes of cubes for different number of incident fields without noise. The number of Rayleigh coefficients measured in each reconstruction is $4\left(M_{1}+M_{2}+1\right)^{2}$. The contrast $q=\left(x_{3}+1\right)\left(\sin \left(x_{1}\right)^{2} \sin \left(x_{2}\right)^{2}+0.3\right) / 4-0.4 \mathrm{i}$ in $D$. (b) 48 propagating modes, 52 evanescent modes, isovalue 40 (c) 52 propagating modes, 312 evanescent modes, isovalue 1.8 (d) 52 propagating modes, 1104 evanescent modes, isovalue 0.008 . 


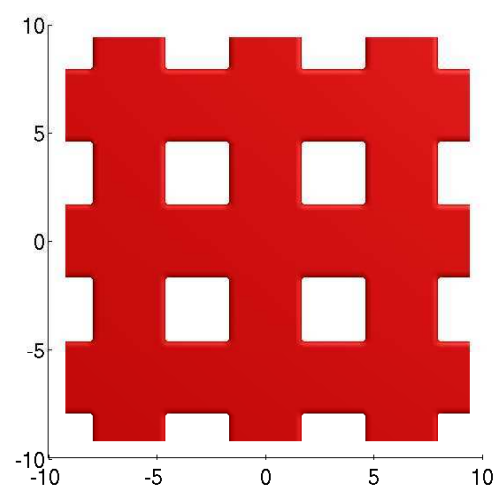

(a) Exact geometry (view down $x_{3}$ axis)

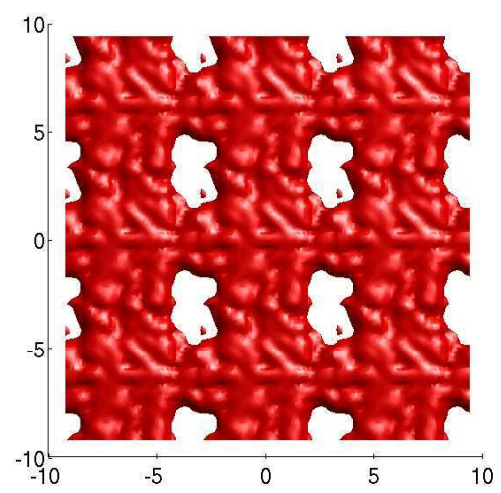

(c) $M_{1,2}=4$ (view down $x_{3}$ axis)

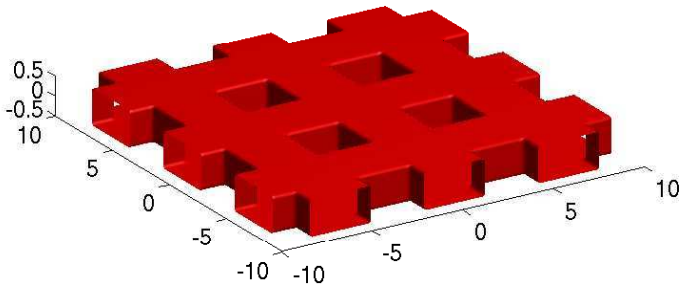

(e) Exact geometry (3D view)

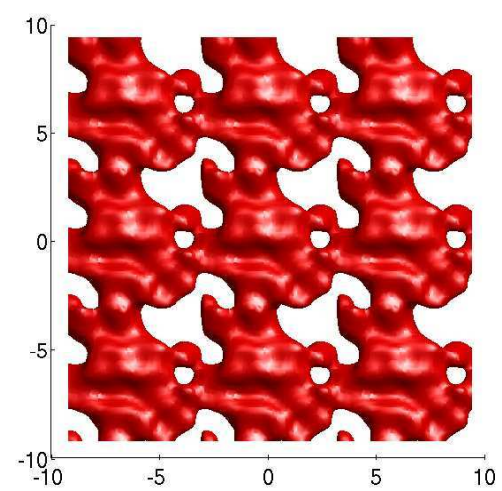

(b) $M_{1,2}=2$ (view down $x_{3}$ axis)

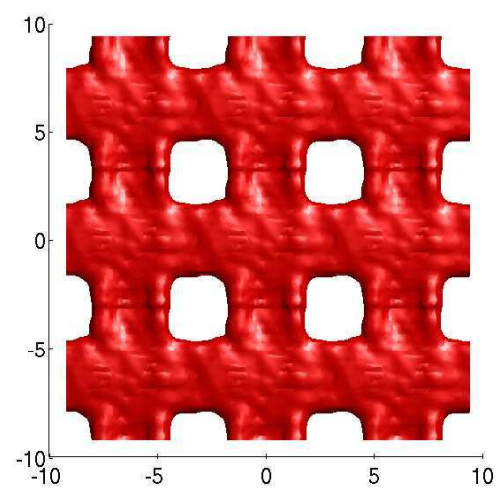

(d) $M_{1,2}=8$ (view down $x_{3}$ axis)

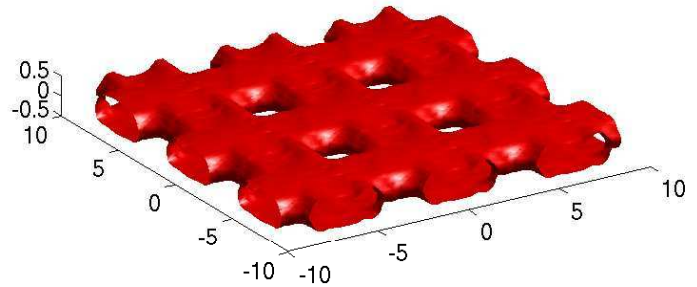

(f) $M_{1,2}=8(3 \mathrm{D}$ view $)$

Figure 3: Reconstructions of biperiodic shapes of plus signs for different number of incident fields without noise. The number of Rayleigh coefficients measured in each reconstruction is $4\left(M_{1}+M_{2}+1\right)^{2}$. The contrast $q=0.5-0.6$ in $D_{1}=\left\{\left(x_{1}, x_{2}\right)^{\top} \in D:-1<x_{1}<1\right\}$ and $q=0.3$ in $D \backslash D_{1}$. (b) 48 propagating modes, 52 evanescent modes, isovalue 15 (c) 52 propagating modes, 312 evanescent modes, isovalue 2 (d) 52 propagating modes, 1104 evanescent modes, isovalue 0.05 . 


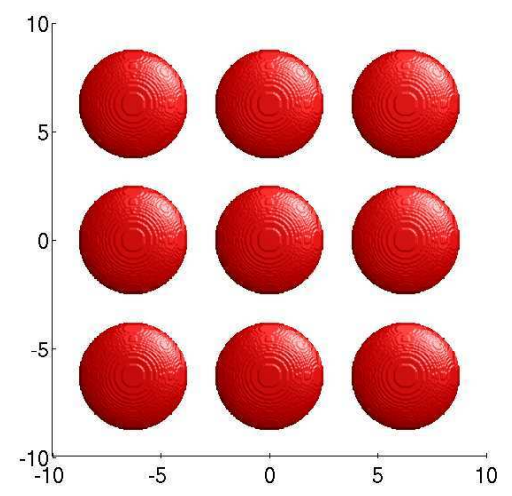

(a) Exact geometry (view down $x_{3}$ axis)

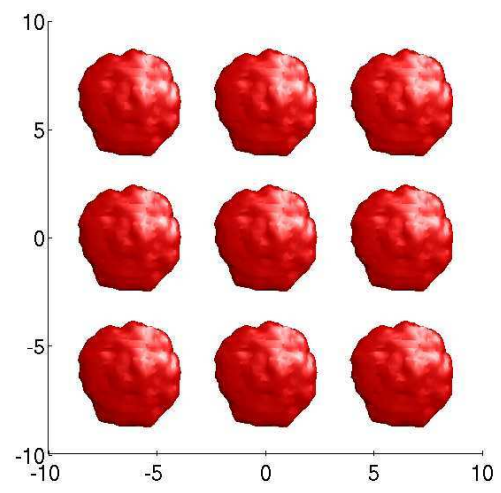

(b) $2 \%$ artificial noise, $M_{1,2}=8$ (view down $x_{3}$ axis)

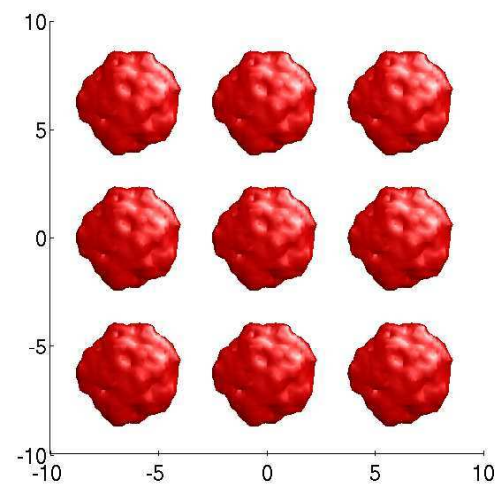

(c) $5 \%$ artificial noise, $M_{1,2}=8$ (view down $x_{3}$ axis)

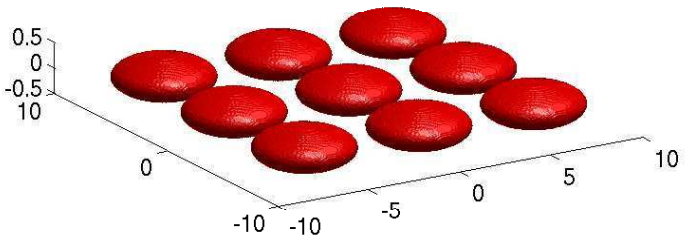

(d) Exact geometry (3D view)

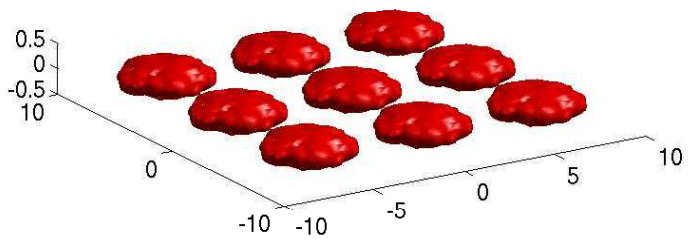

(e) $5 \%$ artificial noise, $M_{1,2}=8$ (3D view)

Figure 4: Reconstructions of biperiodic shapes of ellipsoids for artificial noise. The number of Rayleigh coefficients measured in each reconstruction is $4\left(M_{1}+M_{2}+1\right)^{2}$. The contrast $q=0.5$ in $D$. (b) 52 propagating modes, 1104 evanescent modes, isovalue 0.0012 (c) 52 propagating modes, 1104 evanescent modes, isovalue 0.0023 . 


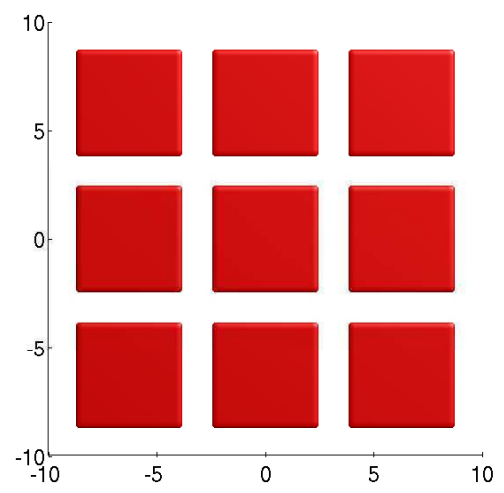

(a) Exact geometry (view down $x_{3}$ axis)

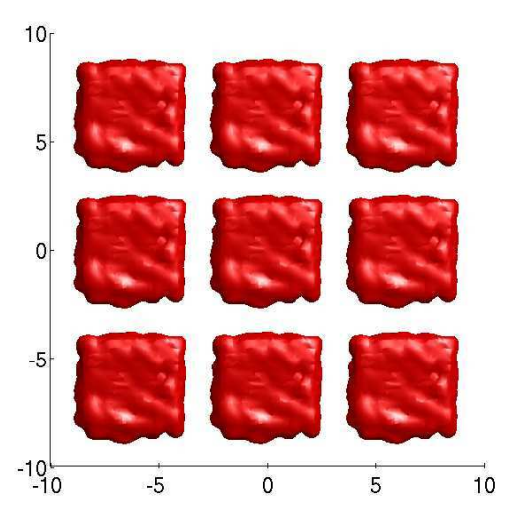

(b) $2 \%$ artificial noise, $M_{1,2}=8$ (view down $x_{3}$ axis)

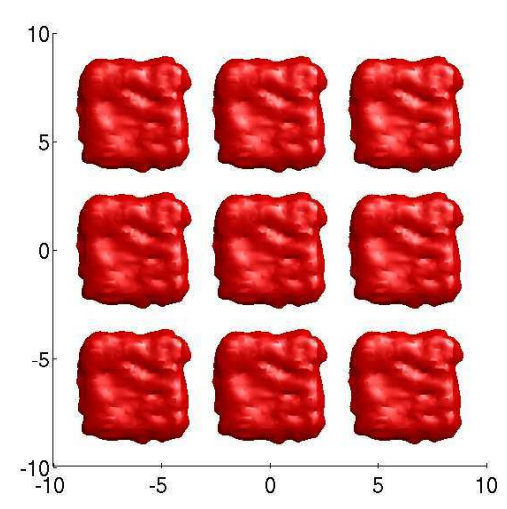

(c) $5 \%$ artificial noise, $M_{1,2}=8$ (view down $x_{3}$ axis)

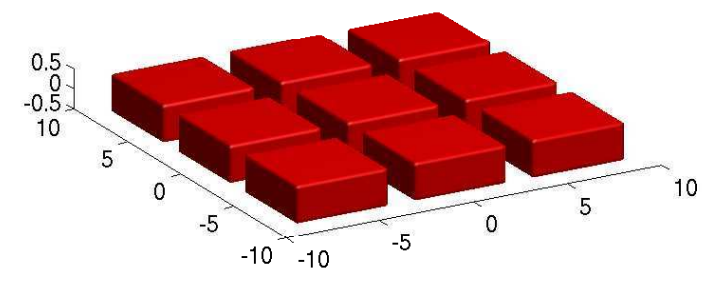

(d) Exact geometry (3D view)

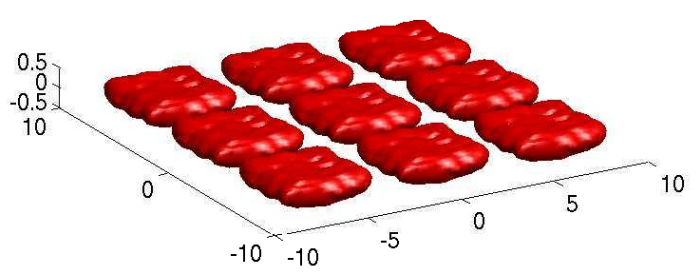

(e) $5 \%$ artificial noise, $M_{1,2}=8$ (3D view)

Figure 5: Reconstructions of biperiodic shapes of cubes for artificial noise. The number of Rayleigh coefficients measured in each reconstruction is $4\left(M_{1}+M_{2}+1\right)^{2}$. The contrast $q=\left(x_{3}+1\right)\left(\sin \left(x_{1}\right)^{2} \sin \left(x_{2}\right)^{2}+\right.$ $0.3) / 4-0.4 \mathrm{i}$ in $D$. (b) 52 propagating modes, 1104 evanescent modes, isovalue 0.1 (c) 52 propagating modes, 1104 evanescent modes, isovalue 0.02 . 


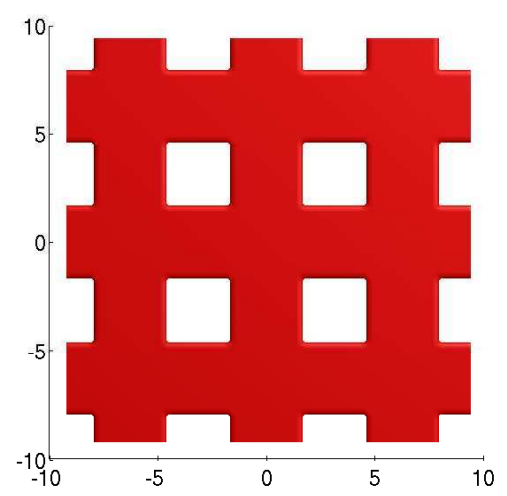

(a) Exact geometry (view down $x_{3}$ axis)

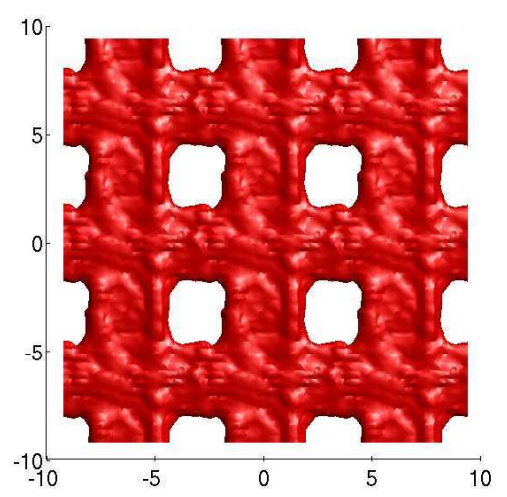

(b) $2 \%$ artificial noise, $M_{1,2}=8$ (view down $x_{3}$ axis)

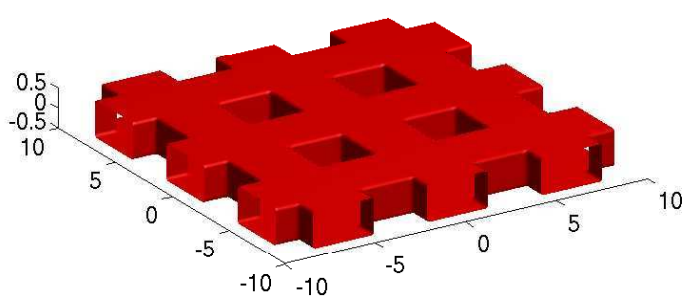

(d) Exact geometry (3D view)

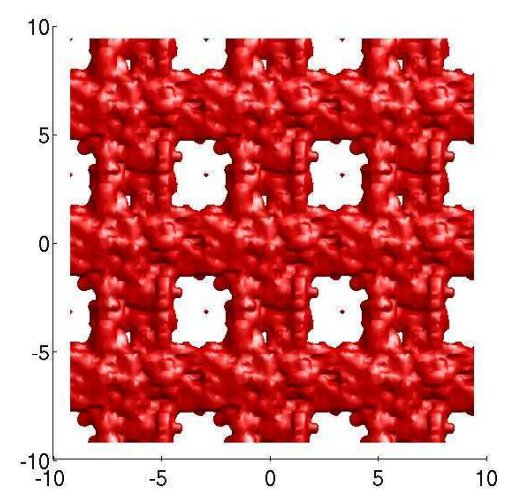

(c) $5 \%$ artificial noise, $M_{1,2}=8$ (view down $x_{3}$ axis)

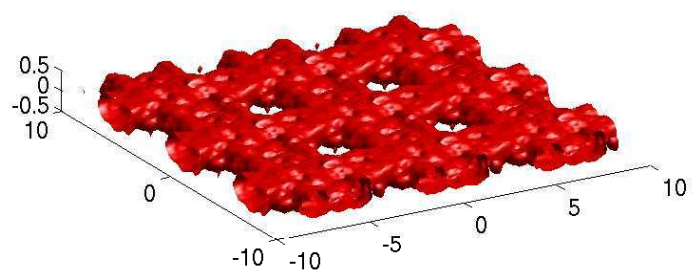

(e) $5 \%$ artificial noise, $M_{1,2}=8$ (3D view)

Figure 6: Reconstructions of biperiodic shapes of plus signs for artificial noise. The number of Rayleigh coefficients measured in each reconstruction is $4\left(M_{1}+M_{2}+1\right)^{2}$. The contrast $q=0.5-0.6 \mathrm{i}$ in $D_{1}=$ $\left\{\left(x_{1}, x_{2}\right)^{\top} \in D:-1<x_{1}<1\right\}$ and $q=0.3$ in $D \backslash D_{1}$. (b) 52 propagating modes, 1104 evanescent modes, isovalue 0.1 (c) 52 propagating modes, 1104 evanescent modes, isovalue 0.02 . 
[14] D. C. Dobson, A variational method for electromagnetic diffraction in biperiodic structures, Math. Model. Numer. Anal., 28 (1994), pp. 419-439.

[15] G. Hu, F. QU, AND B. Zhang, A linear sampling method for inverse problems of diffraction gratings of mixed types, Math. Meth. Appl. Sci., 35 (2012), pp. 1047-1066.

[16] G. Hu AND B. ZhANG, The linear sampling method for the inverse electromagnetic scattering by a partially coated bi-periodic structure, Math. Meth. Appl. Sci., 34 (2011), pp. 509-519.

[17] A. KIRSCH, Uniqueness theorems in inverse scattering theory for periodic structures, Inverse Problems, 10 (1994), pp. 145-152.

[18] - Characterization of the shape of a scattering obstacle using the spectral data of the far field operator, Inverse Problems, 14 (1998), pp. 1489-1512.

[19] — The factorization method for a class of inverse elliptic problems, Math. Nachr., 278 (2004), pp. 258-277.

[20] A. Kirsch and N. Grinberg, The Factorization Method for Inverse Problems, Oxford Lecture Series in Mathematics and its Applications 36, Oxford University Press, 2008.

[21] A. Kirsch And S. RitTer, A linear sampling method for inverse scattering from an open arc, Inverse Problems, 16 (2000), pp. 89-105.

[22] A. LeChleiter, The Factorization method is independent of transmission eigenvalues, Inverse Problems and Imaging, 3 (2009), pp. 123-138.

[23] — Imaging of periodic dielectrics, BIT, 50 (2010), pp. 59-83.

[24] A. Lechleiter And D.-L. NGUyen, A galerkin method for strongly singular volume integral equations arising in grating scattering, Preprint, (2012).

[25] W. McLean, Strongly Elliptic Systems and Boundary Integral Operators, Cambridge University Press, Cambridge, UK, 2000.

[26] P. Monk, Finite Element Methods for Maxwell's Equations, Oxford Science Publications, Oxford, 2003.

[27] W. Rudin, Functional Analysis, McGraw-Hill, 2. ed., 1991.

[28] K. SANDFORT, The factorization method for inverse scattering from periodic inhomogeneous media, $\mathrm{PhD}$ thesis, Karlsruher Institut für Technologie, 2010.

[29] G. Schmidt, On the diffraction by biperiodic anisotropic structures, Appl. Anal., 82 (2003), pp. 75-92.

[30] H. Triebel, Höhere Analysis, VEB Deutscher Verlag der Wissenschaften, Berlin, 1972.

[31] C. WeBer, A local compactness theorem for Maxwell's equations, Math. Methods Appl. Sci., 2 (1980), pp. $12-25$.

[32] J. YAng AND B. Zhang, A inverse transmission scattering problem for periodic media, Inverse Problems, 27 (2011), p. 22pp.

[33] J. YAng, B. Zhang, AND R. Zhang, A sampling method for the inverse transmission problem for periodic media, Inverse Problems, 28 (2012), p. 17pp. 